\title{
BASE SIZES OF PRIMITIVE GROUPS: BOUNDS WITH EXPLICIT CONSTANTS
}

\author{
ZOLTÁN HALASI, MARTIN W. LIEBECK, AND ATTILA MARÓTI
}

\begin{abstract}
We show that the minimal base size $b(G)$ of a finite primitive permutation group $G$ of degree $n$ is at most $2(\log |G| / \log n)+24$. This bound is asymptotically best possible since there exists a sequence of primitive permutation groups $G$ of degrees $n$ such that $b(G)=\lfloor 2(\log |G| / \log n)\rceil-2$ and $b(G)$ is unbounded. As a corollary we show that a primitive permutation group of degree $n$ that does not contain the alternating group $\operatorname{Alt}(n)$ has a base of size at most $\max \{\sqrt{n}, 25\}$.
\end{abstract}

\section{INTRODUCTION}

Let $G$ be a permutation group acting on a finite set $\Omega$ of size $n$. A subset $\Sigma$ of $\Omega$ is called a base for $G$ if the pointwise stabilizer of $\Sigma$ in $G$ is trivial. The minimal size of a base for $G$ on $\Omega$ is denoted by $b_{\Omega}(G)$ or by $b(G)$ in case $\Omega$ is clear from the context.

The minimal base size of a primitive permutation group has been much investigated. Already in the nineteenth century Bochert [6] showed that $b(G) \leq n / 2$ for a primitive permutation group $G$ of degree $n$ not containing $\operatorname{Alt}(n)$. This bound was substantially improved by Babai to $b(G)<4 \sqrt{n} \log n$, for uniprimitive groups $G$, in [2], and to the estimate $b(G)<2^{c \sqrt{\log n}}$ for a universal constant $c$, for doubly transitive groups $G$ not containing $\operatorname{Alt}(n)$, in 3 . (Here and throughout the paper the base of the logarithms is 2 unless otherwise stated.) The latter bound was improved by Pyber [30] to $b(G)<c(\log n)^{2}$ where $c$ is a universal constant. These estimates are elementary in the sense that their proofs do not require the Classification of Finite Simple Groups (CFSG). Using CFSG, Liebeck [23] classified all primitive permutation groups $G$ of degree $n$ with $b(G) \geq 9 \log n$.

It is easy to see that any permutation group $G$ of degree $n$ satisfies $|G|<n^{b(G)}$, and hence $b(G)>\log |G| / \log n$. A well-known question of Pyber [31, Page 207], going back to 1993, asks whether there exists a universal constant $c$ such that $b(G)<c(\log |G| / \log n)$ for all primitive groups $G$. This question generalizes other conjectures in the area: for example, the Cameron-Kantor conjecture, which asserts that every almost simple primitive group in a non-standard action has base size bounded by a universal constant $C$; and Babai's conjecture, that there is a function $f: \mathbb{N} \rightarrow \mathbb{N}$ such that any primitive group that has no alternating or classical composition factor of degree or dimension greater than $d$, has base size less than $f(d)$.

Date: February 21, 2018.

2010 Mathematics Subject Classification. 20B15, 20C99, 20B40.

Key words and phrases. minimal base size, primitive permutation group, classical group, irreducible linear group.

The work of the first and third authors on the project leading to this application has received funding from the European Research Council (ERC) under the European Union's Horizon 2020 research and innovation programme (grant agreement No 741420, 617747, 648017). The first and third authors were supported by the János Bolyai Research Scholarship of the Hungarian Academy of Sciences, were funded by a Humboldt Return Fellowship and also by the National Research, Development and Innovation Office (NKFIH) Grant No. K115799. 
The Cameron-Kantor conjecture was proved in 25 (and in a strong form with $C=7$ in [7], [10]). Babai's conjecture was proved in [17] with $f$ a quadratic function (improved to a linear function in [25]).

Despite a great deal of attention, Pyber's conjecture remained open until very recently, when it was proved in [13]. It is shown in [13] that there exists a universal constant $c>0$ such that for every primitive permutation group $G$ of degree $n$ we have

$$
b(G)<45(\log |G| / \log n)+c .
$$

To obtain a more explicit, usable bound, one would like to reduce the multiplicative constant 45 in the above, and also to estimate the constant $c$.

In this paper we achieve this aim. Our main result is the following.

Theorem 1.1. Let $G$ be a primitive permutation group of degree $n$. Then the minimal base size $b(G)$ satisfies

$$
b(G) \leq 2 \frac{\log |G|}{\log n}+24 .
$$

The multiplicative constant 2 in Theorem 1.1 is best possible, as is shown by the following.

\section{Proposition 1.2.}

(i) For every positive integer $k$ there exists a sequence of finite primitive permutation groups $G_{n}$ of degrees $n$ such that as $n \rightarrow \infty$,

$$
\left(b\left(G_{n}\right) \log n\right) / \log \left|G_{n}\right| \rightarrow 2 k /(k+1) .
$$

(ii) There is an infinite sequence of primitive permutation groups $H_{n}$ of degrees $n$ such that $b\left(H_{n}\right)=\left\lfloor 2\left(\log \left|H_{n}\right| / \log n\right)\right\rceil-2$ for all $n$ and $b\left(H_{n}\right)$ is unbounded.

A corollary of Theorem 1.1 and its proof is the following.

Corollary 1.3. Let $G$ be a primitive permutation group of degree $n$ not containing $\operatorname{Alt}(n)$. Then $G$ has a base of size at most $\max \{\sqrt{n}, 25\}$.

Theorem 1.1 is proved for almost simple groups in the next two sections (see Theorems 2.1, 3.1 and 4.2): alternating and symmetric groups are handled in $\S 2$, and classical groups in $\S 3$. The remaining non-affine primitive groups are covered in $\S 4$ (see Theorem 4.1), and affine groups in $\S 5$ (Theorem 5.1). Proposition 1.2 follows from Proposition 2.6 and Proposition 5.3. Finally, Corollary 1.3 is proved in Section 6 .

\section{Alternating And symmetric groups}

In this section we consider the minimal base sizes of alternating and symmetric groups in primitive actions. Here is the main result.

Theorem 2.1. Let $G$ be a primitive permutation group of degree $n$ with socle isomorphic to $\operatorname{Alt}(m)$ for some integer $m \geq 5$. Then

$$
b(G) \leq 2 \frac{\log |G|}{\log n}+16 .
$$

In the proof of Theorem 2.1, we may assume that $19 \leq b(G) \leq \log |G|$. In particular $m \geq 7$ and $G=\operatorname{Alt}(m)$ or $\operatorname{Sym}(m)$.

Let $\Omega$ be a set of size $n$ permuted by $G$, let $\alpha \in \Omega$ and let $H=G_{\alpha}$, a maximal subgroup of $G$. There are three possiblities to consider, according to the action of $H$ on the underlying set $\{1, \ldots, m\}$ : 
(1) $H$ is intransitive: here $H=(\operatorname{Sym}(k) \times \operatorname{Sym}(m-k)) \cap G$ for some $k \leq m / 2$;

(2) $H$ is transitive and imprimitive: here $H=(\operatorname{Sym}(b) \imath \operatorname{Sym}(a)) \cap G$, where $m=a b$;

(3) $H$ is primitive on $\{1, \ldots, m\}$.

In case (1), the action of $G$ on $\Omega$ is the action on $k$-element subsets of $\{1, \ldots, m\}$, and in case (2) the action is on partitions into $a$ parts of size $b$. These actions are considered in Sections 2.1 and 2.2, and the proof of Theorem 2.1 is completed in Section 2.3.

2.1. Action on subsets. Here we prove Theorem 2.1 in the case when the action is on subsets (see Proposition 2.5). Let $\operatorname{Sym}(m)$ act on the set $\Omega(m, k)$ of all $k$-element subsets of the set $\{1, \ldots, m\}$, where $k \leq m / 2$. Set $n=|\Omega(m, k)|=\left(\begin{array}{c}m \\ k\end{array}\right)$. Let $b(m, k)$ denote the minimal size of a base for $\operatorname{Sym}(m)$ acting on $\Omega(m, k)$. For convenience set $t=m / k$.

A detailed study of the function $b(m, k)$ was carried out in [20]. Here are the main results from that paper that we need.

Theorem 2.2. ([20, Thm. 3.2, Cor. 4.3])

(i) We have $b(m, k) \leq\left\lceil\log _{\lceil t\rceil}(m)\right\rceil(\lceil t\rceil-1)$.

(ii) If $k^{2} \leq m$, then

$$
b(m, k)=\left\lceil\frac{2 m-2}{k+1}\right\rceil<\frac{2 m}{k+1}+1=\frac{2 k}{k+1} t+1 .
$$

We shall need the following estimates for $\ln |\operatorname{Sym}(m)| / \ln |\Omega(m, k)|$.

Lemma 2.3. We have

$$
\left(\frac{t}{\ln (t)+1}\right)(\ln m-1)<\frac{\ln |\operatorname{Sym}(m)|}{\ln |\Omega(m, k)|}<\left(\frac{t}{\ln (t)}\right) \ln m .
$$

Proof. By the inequalities

$$
(m / k)^{k}<\left(\begin{array}{c}
m \\
k
\end{array}\right)<(m e / k)^{k} \text { and }(m / e)^{m}<m !<m^{m},
$$

we have

$$
\frac{m(\ln m-1)}{k(\ln (m / k)+1)}<\frac{\ln |\operatorname{Sym}(m)|}{\ln |\Omega(m, k)|}<\frac{m \ln m}{k \ln (m / k)}=\frac{m / k}{\ln (m / k)} \ln m .
$$

From this the lemma follows.

The next result establishes the conclusion of Theorem 2.1 under the assumption that $k^{2} \leq m$.

Lemma 2.4. Assume that $k^{2} \leq m$. Then

$$
b(m, k)<2 \frac{\ln |\operatorname{Sym}(m)|}{\ln |\Omega(m, k)|}+4 .
$$

Proof. Assume first that $k \geq 8>e^{2}$. By Theorem 2.2(ii) and Lemma 2.3, we have

$$
\frac{b(m, k) \ln n}{\ln |\operatorname{Sym}(m)|}<\left(\frac{2 t+1}{t}\right)\left(\frac{\ln (t)+1}{\ln (m)-1}\right) \text {. }
$$

Since $k \geq 8>e^{2}$, it follows that $\frac{\ln (t)+1}{\ln (m)-1}<1$. By this and Lemma 2.3,

$$
b(m, k)<2 \frac{\ln |\operatorname{Sym}(m)|}{\ln n}+\left(\frac{\ln (m)}{\ln (m)-1}\right)\left(\frac{\ln (t)+1}{\ln (t)}\right) .
$$

It is easy to see that the second term is less than 4 , giving the conclusion in this case $\left(k \geq 8>e^{2}\right)$. 
Hence we may assume that $k \leq 7$. A GAP [15] computation shows that the bound in the conclusion of the lemma holds for $5 \leq m \leq 148<e^{5}$. Thus assume also that $m \geq 149>e^{5}$.

If $2 \leq k \leq 7$ then Theorem 2.2 gives $b(m, k)<\frac{2 k}{k+1} t+1$, and so by Lemma 2.3 ,

$$
\frac{b(m, k) \ln n}{\ln |\operatorname{Sym}(m)|}<\left(\frac{2 k}{k+1}+\frac{k}{m}\right)\left(\frac{\ln m-\ln k+1}{\ln m-1}\right) .
$$

This is less than 2 for $m \geq 149>e^{5}$.

Here is the main result of this subsection.

Proposition 2.5. We have

$$
b(m, k) \leq 2 \frac{\ln |\operatorname{Sym}(m)|}{\ln |\Omega(m, k)|}+16 .
$$

Proof. By Lemma 2.4, we may assume that $k^{2}>m$, which is equivalent to saying that $t^{2}<m$.

Define $r$ to be the integer $r \geq 2$ with $t^{r}<m \leq t^{r+1}$. Then by Theorem 2.2(i), we have $b(m, k) \leq(r+1) t$. By Lemma 2.3, this gives

$$
\frac{b(m, k) \ln n}{\ln m !}<\frac{(r+1)(\ln t+1)}{r \ln t-1} .
$$

A GAP [15] computation shows that the right hand side is less than 2 provided that $r=2$ and $t \geq 149>e^{5}$, or $r=3$ and $t \geq 20>e^{3}$, or $r \geq 4$ and $t \geq 11$.

If $r=3$ and $t \leq 20<e^{3}$, then $4 t-2\left(\frac{3 \ln t-1}{\ln t+1}\right) t \leq 16$, which gives the conclusion (using Lemma 2.3). Similarly, if $r \geq 4$ and $t<11$, then $(r+1) t-2\left(\frac{r \ln t-1}{\ln t+1}\right) t \leq 11$, giving the conclusion.

This leaves the case where $r=2$ and $t \leq 148<e^{5}$. We first distinguish eleven different cases according to some possible ranges of values of $\ln t$. If $\ln t$ falls in any of the intervals $[\epsilon, \epsilon+0.2]$ where $\epsilon=2.8+0.2 \ell$ and $\ell$ is a non-negative integer at most 10 , then $3 t-2\left(\frac{2 \ln t-1}{\ln t+1}\right) t \leq 16$. Thus we may assume that $t<e^{2.8}$. But then $m \leq t^{3}<e^{8.4}<4500$. By a GAP [15] calculation, we see that if $5 \leq m \leq 4500$, then $3 t-2\left(\frac{2 \ln t-1}{\ln t+1}\right) t \leq 11$. This completes the proof.

The final result of this subsection gives the first part of Proposition 1.2 ,

Proposition 2.6. Fix a positive integer $k$. Then as $m \rightarrow \infty$,

$$
\frac{b(m, k) \log |\Omega(m, k)|}{\log |\operatorname{Sym}(m)|} \rightarrow 2 k /(k+1) .
$$

Proof. Assume that $m \geq k^{2}$. Then, by Theorem $\left[2.2(\right.$ ii) $), b(m, k)=\left\lceil\frac{2 m-2}{k+1}\right\rceil$, and hence $b(m, k) / m \rightarrow \frac{2}{k+1}$ as $m \rightarrow \infty$. Also $(m \ln |\Omega(m, k)| / \ln |\operatorname{Sym}(m)|) \rightarrow k$ by Lemma 2.3 . The result follows.

2.2. Action on partitions. Now consider the minimal base size $f(a, b)$ of the group $\operatorname{Sym}(m)$ acting on the set $\Omega$ of all partitions of $\{1, \ldots, m\}$ into $a$ parts each of size $b$, where $m=a b$ and $a, b \geq 2$. In this case $n=|\Omega|=m ! /\left(b !^{a} a !\right)$. Bases for this action were studied in [5], where the following was proved.

Theorem 2.7. ([5]) Suppose $b \geq 3$. Then one of the following holds:

(i) $a \geq b$ and $f(a, b) \leq 6$;

(ii) $a<b$ and $f(a, b) \leq \log _{a}(b)+4$. 
We shall need the following bound.

Lemma 2.8. Let $a, b$ be integers with $2 \leq a<b$. Then

$$
\frac{\ln b}{\ln a}-1<\frac{\ln ((a b) !)}{\ln \left(\frac{(a b) !}{(b !)^{a} a !}\right)}
$$

Proof. Write $g(a, b)=\ln ((a b) !) / \ln \left(\frac{(a b) !}{(b !)^{a} a !}\right)$. Then using the bounds

$$
\sqrt{2 \pi} \cdot \ell^{1 / 2}\left(\frac{\ell}{e}\right)^{\ell}<\ell !<e \cdot \ell^{1 / 2}\left(\frac{\ell}{e}\right)^{\ell}
$$

which hold for all positive integers $\ell$, we have

$$
\begin{aligned}
g(a, b) & >\frac{a b(\ln (a b)-1)}{\ln ((a b) !)-a \ln (b !)-\ln (a !)} \\
& >\frac{a b(\ln (a b)-1)}{\ln (e / \sqrt{2 \pi})+a b(\ln a)+\frac{1}{2} \ln (a b)-a \ln (\sqrt{2 \pi})-\frac{1}{2} a \ln b-\frac{1}{2} \ln a-a \ln a+a} \\
& =\frac{\ln (a b)-1}{\ln a+\frac{1}{b}\left(1-\ln a-\frac{1}{2} \ln (2 \pi)\right)+\ln (e / \sqrt{2 \pi}) /(a b)+\frac{1}{2 b}((\ln (b)) / a-\ln b)} \\
& \geq \frac{\ln (a b)-1}{\ln a+\frac{1}{b}\left(1-\ln a-\frac{1}{2} \ln (2 \pi)-\frac{\ln b}{4}\right)+\ln (e / \sqrt{2 \pi}) /(a b)} \geq \\
& \geq \frac{\ln (a b)-1}{\ln a+\frac{1}{b}\left(1-\ln 2-\frac{1}{2} \ln (2 \pi)-\frac{\ln 3}{4}+\ln (e / \sqrt{2 \pi}) / 2\right)} \\
& >\frac{\ln (a b)-1}{\ln a-(0.8) / b}>\frac{\ln b}{\ln a}-1 .
\end{aligned}
$$

Here is the main result of this subsection.

Proposition 2.9. With the above notation, we have $f(a, b) \leq \frac{\ln |\operatorname{Sym}(m)|}{\ln n}+5$.

Proof. If $b \geq 3$, this follows immediately from Theorem 2.7. And for $b=2$, Remark 1.6(ii) of [8] gives $f(a, 2) \leq 3$.

2.3. Proof of Theorem 2.1. Let $G=\operatorname{Alt}(m)$ or $\operatorname{Sym}(m)$ act primitively on a set $\Omega$, and let $H$ be a point-stabilizer in $G$. The cases where $\Omega$ is a set of $k$-subsets or partitions of $\{1, \ldots, m\}$ have been dealt with in Propositions 2.5 and 2.9. Hence by the remarks at the beginning of the section, we may assume that $H$ is primitive on $\{1, \ldots, m\}$. In this case, it is proved in [8, Cor. 2] that $b_{\Omega}(G) \leq 5$. This completes the proof of Theorem 2.1.

\section{Classical groups}

In this section we study base sizes of primitive actions of classical groups. Our main result is the following.

Theorem 3.1. Let $G$ be an almost simple primitive permutation group of degree $n$ whose socle is a classical simple group. Then $b(G) \leq 2(\log |G| / \log n)+16$.

We shall divide the proof of this theorem into several subcases. First we give a definition, taken from [25]. Let $G$ be an almost simple group with socle $G_{0}$, a classical group with natural module $V$, a vector space of dimension $d$ over a field $\mathbb{F}_{q}$ of characteristic $p$. We call a maximal subgroup $M$ of $G$ a subspace subgroup if it is reducible on $V$, or is an orthogonal group on $V$ embedded in a symplectic group with $p=2$; more specifically, $M$ is a subspace subgroup if one of the following holds:

(1) $M=G_{U}$ for some proper nonzero subspace $U$ of $V$, where $U$ is totally singular, non-degenerate, or, if $G$ is orthogonal and $p=2$, a nonsingular 1-space ( $U$ is any subspace if $G_{0}=P S L(V)$ ); 
(2) $G_{0}=P S L(V), G$ contains a graph automorphism of $G_{0}$, and $M \cap G_{0}=\left(G_{0}\right)_{U, W}$ where $U, W$ are proper nonzero subspaces of $V, \operatorname{dim} V=\operatorname{dim} U+\operatorname{dim} W$ and either $U \subseteq W$ or $V=U \oplus W$

(3) $G_{0}=S p_{2 m}(q), p=2$ and $M \cap G_{0}=O_{2 m}^{ \pm}(q)$.

Note that in (3), if we regard $G_{0}$ as the isomorphic orthogonal group $O_{2 m+1}(q)$, then $M \cap G_{0}=O_{2 m}^{ \pm}(q)$ is the stabilizer of a hyperplane of the natural module of dimension $2 m+1$.

If $M$ is a subspace subgroup, we call the action of $G$ on the coset space $G / M$ a subspace action.

Bases for non-subspace actions of classical groups were studied in detail in [7, so our main task is to prove Theorem 3.1 for subspace actions. First we require the following general bound.

Proposition 3.2. Let $G$ be as above, and suppose $M$ is as in (1), so that the coset space $X=G / M$ is a $G$-orbit of $k$-dimensional subspaces of $V$, for some $k$. Assume also that $k \leq d / 2$. Then

$$
\frac{\log |G|}{\log |X|} \geq \frac{d}{t k}-1
$$

where $t=1$ if $G_{0}=P S L(V)$, and $t=2$ otherwise.

Proof. Observe that $|G|>q^{\left(d^{2} / t\right)-d}$, while

$$
|X| \leq\left(\begin{array}{l}
d \\
k
\end{array}\right)_{q}:=\frac{\left(q^{d}-1\right)\left(q^{d}-q\right) \cdots\left(q^{d}-q^{k-1}\right)}{\left(q^{k}-1\right)\left(q^{k}-q\right) \cdots\left(q^{k}-q^{k-1}\right)} \leq\left(\frac{q^{d}}{q^{k-1}}\right)^{k}=q^{d k-k^{2}+k} .
$$

Hence

$$
\frac{\log |G|}{\log |X|} \geq \frac{\left(d^{2} / t\right)-d}{d k-k^{2}+k} \geq \frac{d}{t k}-1
$$

3.1. Action on an orbit of subspaces. In this subsection we prove Theorem 3.1 for subspace actions of classical simple groups as in case (1) in the list above. This is the main part of the proof of the theorem.

Theorem 3.3. Let $G$ be a simple classical group on $V$, a vector space of dimension d over $\mathbb{F}_{q}$. Let $X$ be a $G$-orbit of $k$-dimensional subspaces of $V$ with $k \leq d / 2$, on which $G$ acts primitively. Then

$$
b_{X}(G) \leq \frac{d}{k}+11 .
$$

Proof. In every subcase, we will define a base $\mathcal{B} \subset X$ of $G$ in a number of steps. We do this by starting with $\mathcal{B}=\emptyset$ and at each step adding some subspaces to $\mathcal{B}$. Throughout the proof, $G_{(\mathcal{B})}$ denotes the pointwise stabilizer of $\mathcal{B}$ - that is, the set of group elements that fix all the subspaces in $\mathcal{B}$.

Action on the set of all $k$-dimensional subspaces.

First, let us assume that $X$ is the set of all $k$-dimensional subspaces. Let $d=a k+r$ for $a \geq 2$ and $0 \leq r<k$. Take any direct sum decomposition $V=V_{1} \oplus \ldots \oplus V_{a} \oplus U$ with $\operatorname{dim} V_{i}=k$ for $1 \leq i \leq a$, and let $V_{1}, \ldots, V_{a} \in \mathcal{B}$. Fix a basis $B_{i}=\left\{x_{1}^{(i)}, \ldots, x_{k}^{(i)}\right\} \in V_{i}$ for each $i$ and define $W_{1}=\left\langle\sum_{i=1}^{a} x_{s}^{(i)} \mid 1 \leq s \leq k\right\rangle \in X$ and put $W_{1}$ into $\mathcal{B}$. Then the matrix form of the restriction of a $g \in G_{(\mathcal{B})}$ to $V_{1} \oplus \ldots \oplus V_{a}$ is a block diagonal matrix with equal blocks (with respect to the basis $B_{1} \cup \ldots \cup B_{a}$ ). Define $M_{g}=\left[g_{V_{1}}\right]_{B_{1}}=\left[g_{V_{2}}\right]_{B_{2}}=\ldots=$ 
$\left[g_{V_{a}}\right]_{B_{a}}$ and let $\{\gamma, \delta\} \subset S L\left(V_{2}\right)$ be a generating set of $S L\left(V_{2}\right)$ and $C=[\gamma]_{B_{2}}, D=[\delta]_{B_{2}}$ be their matrix forms. Then $g \in G_{(\mathcal{B})}$ fixes the subspaces

$$
W_{2}=\left\langle x_{s}^{(1)}+\gamma\left(x_{s}^{(2)}\right) \mid 1 \leq s \leq k\right\rangle \in X, \quad W_{3}=\left\langle x_{s}^{(1)}+\delta\left(x_{s}^{(2)}\right) \mid 1 \leq s \leq k\right\rangle \in X
$$

if and only if $M_{g}$ commutes with both $C$ and $D$. Thus, putting $W_{2}$ and $W_{3}$ into $\mathcal{B}$, it follows that $G_{(\mathcal{B})}$ acts as scalars on $V_{1} \oplus \ldots \oplus V_{a}$. Finally, if $r>0$ then let $\left\{f_{1}, \ldots, f_{r}\right\}$ be a basis of $U$ and define

$$
W_{4}=\left\langle f_{1}, \ldots, f_{r}, x_{r+1}^{(1)}, \ldots, x_{k}^{(1)}\right\rangle, \quad W_{5}=\left\langle f_{1}+x_{1}^{(2)}, \ldots, f_{r}+x_{r}^{(2)}, x_{r+1}^{(2)}, \ldots, x_{k}^{(2)}\right\rangle .
$$

Adding $W_{4}$ and $W_{5}$ to $\mathcal{B}$ it is easy to see that $G_{(\mathcal{B})}$ contains only scalar transformations. Thus, $b_{X}(G) \leq a+5 \leq \frac{d}{k}+5$ for this case.

Action on an orbit of non-degenerate subspaces.

Now we turn to the case when $G$ is a group fixing some non-degenerate form [, ] on $V$ and $X$ is a $G$-orbit of non-degenerate subspaces. In the special case $d=2 k$, we also assume that the Witt index of elements of $X$ is no more than the Witt index of elements of $X^{\perp}$ (this is only interesting in the orthogonal case, when $k$ is even and $V$ has Witt index $k-1$ ). This will guarantee that the sums defining $u_{i}$ and $v_{i}$ below will have at least two terms.

Let $d=a k+r$ with $1 \leq r \leq k$ and take any orthogonal decomposition $V=V_{1} \oplus$ $\ldots \oplus V_{a} \oplus V_{a+1}$ with $V_{1}, \ldots, V_{a} \in X$. Put $V_{1}, \ldots, V_{a}$ into $\mathcal{B}$. Then any $g \in G_{(\mathcal{B})}$ also fixes $V_{a+1}=\left(\sum_{i=1}^{a} V_{i}\right)^{\perp}$.

Let $l$ and $m \leq 2$ denote the Witt index and the Witt defect of the subspaces in $X$, so $k=2 l+m$. First, let us assume that $l \geq 1$. Then for every $1 \leq s \leq a$, the subspace $V_{s}$ is a direct sum of orthogonal subspaces $V_{s}=V_{s}^{(h)} \oplus V_{s}^{(m)}$, where each $V_{s}^{(h)}$ contains a basis $B_{s}=\left\{x_{1}^{(s)}, \ldots, x_{l}^{(s)}, y_{1}^{(s)}, \ldots, y_{l}^{(s)}\right\}$ with $\left[x_{i}^{(s)}, x_{j}^{(s)}\right]=\left[y_{i}^{(s)}, y_{j}^{(s)}\right]=0,\left[x_{i}^{(s)}, y_{j}^{(s)}\right]=$ $\delta_{i j}$ for all $i, j$ and $V_{s}^{(m)}$ has dimension and Witt defect $m$. (For orthogonal groups of characteristic 2, we also have $Q\left(x_{i}^{(s)}\right)=Q\left(y_{i}^{(s)}\right)=0$ for all $i$, where $Q$ is the underlying quadratic form.) Furthermore, let $l^{\prime}$ be the minimum of $l$ and the Witt index of $V_{a+1}$ and $m^{\prime}=\operatorname{dim}\left(V_{a+1}\right)-2 l^{\prime}$. Similarly to the above, choose an orthogonal decomposition $V_{a+1}=V_{a+1}^{(h)} \oplus V_{a+1}^{(m)}$ along with a basis $B_{a+1}=\left\{x_{1}^{(a+1)}, \ldots, x_{l^{\prime}}^{(a+1)}, y_{1}^{(a+1)}, \ldots, y_{l^{\prime}}^{(a+1)}\right\}$ of $V_{a+1}^{(h)}$ satisfying $\left[x_{i}^{(a+1)}, x_{j}^{(a+1)}\right]=\left[y_{i}^{(a+1)}, y_{j}^{(a+1)}\right]=0,\left[x_{i}^{(a+1)}, y_{j}^{(a+1)}\right]=\delta_{i j}$ for $1 \leq i, j \leq l^{\prime}$, and define $x_{i}^{(a+1)}=y_{i}^{(a+1)}=0$ for $l^{\prime}<i \leq l$.

For $1 \leq i \leq l$, define

$$
u_{i}=\sum_{s=1}^{a+1} x_{i}^{(s)}, v_{i}=\sum_{s=1}^{(a+1)} y_{i}^{(s)}
$$

We define the subspaces

$$
\begin{array}{ll}
W_{1}^{(h)}=\left\langle u_{1}, \ldots, u_{l}, y_{1}^{(1)}, \ldots, y_{l}^{(1)}\right\rangle, & W_{2}^{(h)}=\left\langle x_{1}^{(1)}, \ldots, x_{l}^{(1)}, v_{1}, \ldots, v_{l}\right\rangle, \\
W_{3}^{(h)}=\left\langle u_{1}, \ldots, u_{l}, y_{1}^{(2)}, \ldots, y_{l}^{(2)}\right\rangle, & W_{4}^{(h)}=\left\langle x_{1}^{(2)}, \ldots, x_{l}^{(2)}, v_{1}, \ldots, v_{l}\right\rangle .
\end{array}
$$

Then $W_{t}:=W_{t}^{(h)} \oplus V_{1}^{(m)} \in X$ for each $1 \leq t \leq 4$. Adding $W_{1}, W_{2}, W_{3}, W_{4}$ to $\mathcal{B}$, we see that any $g \in G_{(\mathcal{B})}$ fixes each $V_{s}^{(h)}$ and, moreover, the matrix form of each restriction $g_{V_{s}^{(h)}}$ satisfies

$$
\left[g_{V_{1}^{(h)}}\right]_{B_{1}}=\left[g_{V_{2}^{(h)}}\right]_{B_{2}}=\ldots=\left[g_{V_{a}^{(h)}}\right]_{B_{a}}=\left(\begin{array}{cc}
A_{g} & 0 \\
0 & B_{g}
\end{array}\right)
$$


for some $A_{g}, B_{g} \in G L(l, q)$. The use of the $x_{i}^{(a+1)}, y_{i}^{(a+1)}$ as summands in the $u_{i}$ and $v_{i}$ also implies that

$$
\left[g_{V_{a+1}^{(h)}}\right]_{B_{a+1}}=\left(\begin{array}{cc}
A_{g}^{\prime} & 0 \\
0 & B_{g}^{\prime}
\end{array}\right)
$$

where $A_{g}^{\prime}$ and $B_{g}^{\prime}$ are left upper $l^{\prime} \times l^{\prime}$ submatrices of $A_{g}$ and $B_{g}$, respectively.

Adding also the subspace $W_{5}=W_{5}^{(h)} \oplus V_{1}^{(m)}$ with $W_{5}^{(h)}:=\left\langle x_{i}^{(1)}, y_{i}^{(1)}+x_{i}^{(2)} \mid 1 \leq i \leq l\right\rangle$ we can also guarantee that $A_{g}=B_{g}$ holds for any $g \in G_{(\mathcal{B})}$.

Let $B_{2}^{(x)}=\left\{x_{1}^{(2)}, \ldots, x_{l}^{(2)}\right\}$ and $V_{2}^{(x)}$ be the subspace spanned by $B_{2}^{(x)}$. Choose $\varphi, \psi \in$ $\operatorname{End}\left(V_{2}^{(x)}\right)$ generating $\operatorname{End}\left(V_{2}^{(x)}\right)$ as an algebra. Define

$$
W_{6}^{(h)}=\left\langle x_{i}^{(1)}+\varphi\left(x_{i}^{(2)}\right), y_{i}^{(1)} \mid 1 \leq i \leq l\right\rangle, \quad W_{7}^{(h)}=\left\langle x_{i}^{(1)}+\psi\left(x_{i}^{(2)}\right), y_{i}^{(1)} \mid 1 \leq i \leq l\right\rangle .
$$

and let $W_{6}:=W_{6}^{(h)} \oplus V_{1}^{(m)}, W_{7}:=W_{7}^{(h)} \oplus V_{1}^{(m)}$. Adding $W_{6}, W_{7}$ to $\mathcal{B}$, we see that $g_{V_{2}^{(x)}}$ commutes with both $\varphi$ and $\psi$ for any $g \in G_{(\mathcal{B})}$. Thus $g_{V_{2}^{(x)}}$ is a scalar transformation. Therefore, any $g \in G_{(\mathcal{B})}$ acts as a scalar on $V_{1}^{(h)} \oplus \ldots \oplus V_{a+1}^{(h)}$.

For every $1 \leq s \leq a+1$, choose other orthogonal decompositions $V_{s}=V_{s}^{(h)^{\prime}} \oplus V_{s}^{(m)^{\prime}}$ such that each $V_{s}^{(h)^{\prime}}$ is isometric to $V_{s}^{(h)}$ and $V_{s}^{(h)}+V_{s}^{(h)^{\prime}}=V_{s}$. Applying similar constructions as for $W_{1}, \ldots, W_{4}$ before, by adding 4 further subspaces to $\mathcal{B}$ we can synchronize the action of any $g \in G_{(\mathcal{B})}$ on each $V_{s}^{(h)^{\prime}}$ with its action on each $V_{t}^{(h)}$. Thus, now each $g \in G_{(\mathcal{B})}$ acts as a scalar on the whole vector space $V_{1} \oplus \ldots \oplus V_{a+1}$. Thus, $b_{X}(G) \leq a+7+4 \leq \frac{d}{k}+11$ in this case.

Now, let us assume that $l=0$, so $k=m \leq 2$. The case $k=1$ is trivial. The case $k=m=2$ implies that $V$ is orthogonal. We can also assume that $a \geq 3$, since otherwise $d \leq 6$. Then each $V_{s}$ has a basis $x^{(s)}, y^{(s)}$ with $Q\left(x^{(s)}\right)=1, Q\left(y^{(s)}\right)=\alpha,\left[x^{(s)}, y^{(s)}\right]=1$, where $\alpha \in \mathbb{F}_{q}$ is such that the polynomial $t^{2}+t+\alpha$ is irreducible over $\mathbb{F}_{q}$. Additionally, choose an arbitrary spanning set $\left\{x^{a+1}, y^{a+1}\right\}$ of $V_{a+1}$. Since $\left\{Q(z) \mid z \in V_{s}\right\}=\mathbb{F}_{q}$, we can define

$$
u_{1}=\sum_{s=2}^{a+1} x^{(s)}+z^{(1)}, \quad v_{1}=\sum_{s=2}^{a+1} y^{(s)}+w^{(1)}, \quad u_{2}=\sum_{s=1}^{a-1} x^{(s)}+z^{(a)}, \quad v_{2}=\sum_{s=1}^{a-1} y^{(s)}+w^{(a)}
$$

with $z^{(1)}, w^{(1)} \in V_{1}, z^{(a)}, w^{(a)} \in V_{a}$ such that $Q\left(u_{1}\right)=Q\left(u_{2}\right)=1, Q\left(v_{1}\right)=Q\left(v_{2}\right)=\alpha$. Now, let

$$
\begin{array}{llll}
W_{1}=\left\langle u_{1}, y^{(2)}\right\rangle, & W_{2}=\left\langle u_{1}, y^{(3)}\right\rangle, & W_{3}=\left\langle x^{(2)}, v_{1}\right\rangle, & W_{4}=\left\langle x^{(3)}, v_{1}\right\rangle, \\
W_{5}=\left\langle u_{2}, y^{(1)}\right\rangle, & W_{6}=\left\langle u_{2}, y^{(2)}\right\rangle, & W_{7}=\left\langle x^{(1)}, v_{2}\right\rangle, & W_{8}=\left\langle x^{(2)}, v_{2}\right\rangle .
\end{array}
$$

Adding each $W_{i}$ to $\mathcal{B}$ we see that the restriction of any $g \in G_{(\mathcal{B})}$ to the subspaces $V_{1}, \ldots, V_{a}$ has matrix form

$$
\left[g_{V_{1}}\right]_{\left\{x^{(1)}, y^{(1)}\right\}}=\ldots=\left[g_{V_{a}}\right]_{\left\{x^{(a)}, y^{(a)}\right\}}=\left(\begin{array}{cc}
c & 0 \\
0 & d
\end{array}\right) \text { for some } c, d \in \mathbb{F}_{q} .
$$

Using that $Q\left(g\left(x^{s}\right)\right)=1, Q\left(g\left(y^{(s)}\right)\right)=\alpha,\left[g\left(x^{(s)}, g\left(y^{(s)}\right)\right]=1\right.$ it follows that $c=m= \pm 1$, so any $g \in G_{(\mathcal{B})}$ acts on $V_{1} \oplus \ldots \oplus V_{a}$ as a scalar transformation. The use of $x^{(a+1)}, y^{(a+1)}$ guarantees that $g \in G_{(\mathcal{B})}$ is a scalar on the whole of $V$. Thus, $b_{X}(G) \leq \frac{d}{k}+8$ for this case.

Action on an orbit of totally singular subspaces.

From now on, let $X$ be the set of $k$-dimensional totally singular subspaces of $V$. Again, we can assume that $k \geq 2$. 
Let $l$ be the Witt index and let $m \leq 2$ be the Witt defect of $V$, so $k \leq l$ (since otherwise $X=\emptyset)$ and $d=2 l+m$. Let $l=a k+r$ for $0 \leq r<k$ and denote $w(s)=k$ for $1 \leq s \leq k$ and $w(a+1)=r$. Take an orthogonal decomposition $V=V_{1} \oplus \ldots \oplus V_{a} \oplus V_{a+1} \oplus U$ such that $V_{s}$ has dimension $2 w(s)$ and Witt index $w(s)$ for each $1 \leq s \leq a+1$. For every $1 \leq s \leq a+1$ let $B_{s}=\left\{x_{1}^{(s)}, \ldots, x_{w(s)}^{(s)}, y_{1}^{(s)}, \ldots, y_{w(s)}^{(s)}\right\}$ be a basis of $V_{s}$ such that $V_{s}^{(x)}=\left\langle x_{1}^{(s)}, \ldots, x_{w(s)}^{(s)}\right\rangle, V_{s}^{(y)}=\left\langle y_{1}^{(s)}, \ldots, y_{w(s)}^{(s)}\right\rangle$ are $w(s)$-dimensional singular subspaces, and $\left[x_{i}^{(s)}, y_{j}^{(s)}\right]=\delta_{i j}$ for every $1 \leq i, j \leq w(s)$. Furthermore, define $x_{i}^{(a+1)}=y_{i}^{(a+1)}=0$ for $r<i \leq k$. Finally, take the additional $k$-dimensional singular subspaces

$$
\begin{aligned}
V_{a+1}^{(x)^{\prime}} & =\left\langle x_{1}^{(a+1)}, \ldots, x_{r}^{(a+1)}, x_{r+1}^{(1)}, \ldots, x_{k}^{(1)}\right\rangle, \\
V_{a+1}^{(y)^{\prime}} & =\left\langle y_{1}^{(a+1)}, \ldots, y_{r}^{(a+1)}, y_{r+1}^{(1)}, \ldots, y_{k}^{(1)}\right\rangle .
\end{aligned}
$$

Let $u_{i}=\sum_{s=1}^{(a+1)} x_{i}^{(s)}, v_{i}=\sum_{s=1}^{(a+1)} y_{i}^{(s)}$ for $1 \leq i \leq k$ and define

$$
W_{1}=\left\langle u_{1}, \ldots, u_{k}\right\rangle, \quad W_{2}=\left\langle v_{1}, \ldots, v_{k}\right\rangle .
$$

First, add each of the subspaces $V_{1}^{(x)}, V_{1}^{(y)}, \ldots, V_{a}^{(x)}, V_{a}^{(y)}, V_{a+1}^{(x)^{\prime}}, V_{a+1}^{(y)^{\prime}}, W_{1}, W_{2}$ to $\mathcal{B}$. Then the subspaces $V_{s}$ for each $1 \leq s \leq a+1$ are fixed by any $g \in G_{(\mathcal{B})}$ and the restrictions $g_{V_{s}}$ have matrix form

$$
\begin{gathered}
{\left[g_{V_{1}}\right]_{B_{1}}=\ldots=\left[g_{V_{a}}\right]_{B_{a}}=\left(\begin{array}{cc}
A_{g} & 0 \\
0 & \left(A_{g}\right)^{-T}
\end{array}\right),} \\
{\left[g_{V_{a+1}}\right]_{B_{a+1}}=\left(\begin{array}{cc}
A_{g}^{\prime} & 0 \\
0 & \left(A_{g}^{\prime}\right)^{-T}
\end{array}\right),}
\end{gathered}
$$

where $A_{g} \in G L(k, q)$ and $A_{g}^{\prime}$ is the left upper $r \times r$ submatrix of $A_{g}$.

Next, we define additional $k$-dimensional singular subspaces of the form

$$
\begin{aligned}
& W^{(x)}(C)=\left\langle\sum_{s=1}^{a+1}\left(x_{j}^{(s)}+\sum_{i=1}^{k} c_{i j} y_{i}^{(s)}\right) \mid 1 \leq j \leq k\right\rangle, \\
& W^{(y)}(C)=\left\langle\sum_{s=1}^{a+1}\left(y_{j}^{(s)}+\sum_{i=1}^{k} c_{i j} x_{j}^{(s)}\right) \mid 1 \leq j \leq k\right\rangle,
\end{aligned}
$$

where $C=\left(c_{i j}\right) \in M(k, q)$. The subspaces $W^{(x)}(C)$ and $W^{(y)}(C)$ are singular if the matrix $C$ is symmetric (when $V$ is a symplectic space) or anti-symmetric (when $V$ is an orthogonal or a unitary space). Furthermore, $g \in G_{(\mathcal{B})}$ fixes $W^{(x)}(C)\left(\right.$ resp. $\left.W^{(y)}(C)\right)$ if and only if $A_{g}^{T} C=C A_{g}^{-1}$ (resp. $A_{g} C=C A_{g}^{-T}$ ) holds.

First, let us assume that $V$ is a symplectic space and choose $C, D \in M(k, q)$ symmetric matrices, which generate the full matrix algebra $M(k, q)$ (as an algebra). Adding $W^{(y)}(I), W^{(y)}(C), W^{(y)}(D)$ to $\mathcal{B}$ we see that any $g \in G_{(\mathcal{B})}$ satisfies $A_{g}=A_{g}^{-T}$, and, therefore, $A_{g} C=C A_{g}, A_{g} D=D A_{g}$. It follows that $A_{g}$ is a scalar matrix for any $g \in G_{(\mathcal{B})}$. Thus, $g$ acts as a scalar on the whole $V=V_{1} \oplus \ldots \oplus V_{a} \oplus V_{a+1}$.

Now, let $V$ be an orthogonal or unitary space and choose antisymmetric matrices $C=$ $E_{12}-E_{21}, D=\sum_{i=2}^{k-1}\left(E_{i, i+1}-E_{i+1, i}\right)$. (Here $\left\{E_{i j} \mid 1 \leq i, j \leq k\right\}$ denotes the usual basis of the full matrix algebra $M(k, q)$.) Add the subspaces $W^{(x)}(C), W^{(y)}(C), W^{(x)}(D), W^{(y)}(D)$ to $\mathcal{B}$ and let $g \in G_{(\mathcal{B})}$. Then we have $A_{g} C A_{g}^{T}=A_{g}^{T} C A_{g}=C$ and $A_{g} D A_{g}^{T}=A_{g}^{T} D A_{g}=D$. Using the implication

$$
A X A^{T}=X, A^{T} Y A=Y \Rightarrow A X Y=A X A^{T} Y A=X Y A,
$$

we see that $A_{g}$ commutes with every product $P$ of $C$ 's and $D$ 's with an even number of terms. Similarly, $A_{g} P A_{g}^{T}=A_{g}^{T} P A_{g}=P$ holds for every product $P$ of $C$ 's and $D$ 's with an odd number of terms. In particular, $A_{g}$ commutes with $C D=E_{13},-D C=$ 
$E_{31},-C D^{2} C=E_{11}$ etc. Continuing this way, we see that $A_{g}=\lambda \cdot I$ is a scalar matrix. The equation $A_{g} C A_{g}^{T}=C$ also shows that $\lambda^{2}=1$ and so $A_{g}=A_{g}^{-T}= \pm I$. Thus, any $g \in G_{(\mathcal{B})}$ is a scalar transformation on $V_{1} \oplus \ldots \oplus V_{a+1}$. If $U \neq 0$, we can choose a $k$-dimensional singular subspace $V_{a+2}^{(x)} \leq V_{1} \oplus U$ satisfying $V_{1}+V_{a+2}^{(x)}=V_{1}+U$. Let $x_{1}^{(a+2)}, \ldots, x_{k}^{(a+2)}$ be any basis of $V_{a+2}^{(x)}$. Adding the subspaces $V_{a+2}^{(x)},\left\langle x_{1}^{(a+2)}+y_{1}^{(1)}, \ldots, x_{k}^{(a+2)}+y_{k}^{(1)}\right\rangle$ to $\mathcal{B}$ gives the result. So, $b_{X}(G) \leq 2 a+10 \leq \frac{d}{k}+10$.

The above argument works if $X$ is the set of all totally singular subspaces, which is indeed a $G$-orbit in most cases. The only exception is when $V$ is an orthogonal space, $d=2 k$, and $G=\Omega^{+}(V)$, so we assume this from now on. Then two totally singular $k$-dimensional subspaces $V_{1}, V_{2}$ are in the same $G$-orbit if and only if $\operatorname{dim}\left(V_{1} \cap V_{2}\right) \equiv k$ $(\bmod 2)$. Since the full orthogonal group $O(V)$ interchanges the two $G$-orbits, it does not matter, which orbit we choose. Note that in the above construction the subspaces $V_{1}^{(x)}, V_{1}^{(y)}, W^{(x)}(C), W^{(y)}(C), W^{(x)}(D), W^{(y)}(D)$ are in the same $G$-orbit provided that $k$ is even (the further subspaces defined in the proof are now meaningless). So, $b_{X}(G) \leq 6$ in this case. Now, let $k$ be odd, and choose an orthogonal decomposition $V=\langle x, y\rangle \oplus U$, where $x, y$ is a hyperbolic pair. Then $\operatorname{dim} U=d-2=2(k-1)$, so the above construction works for a $G_{U}$-orbit of $(k-1)$-dimensional totally singular subspaces of $U$, since $k-1$ is even. That is, there are 6 totally singular $(k-1)$-dimensional subspaces $U_{1}, \ldots, U_{6}$ of $U$, which form a base for the action of $G_{U}$ on $U$. By construction,

$$
U_{1}=\left\langle x_{1}, \ldots, x_{k-1}\right\rangle, \quad U_{2}=\left\langle y_{1}, \ldots, y_{k-1}\right\rangle
$$

with $\left[x_{i}, y_{j}\right]=\delta_{i j}$ for $1 \leq i, j \leq k-1$. Define the subspaces $V_{s}=\langle x\rangle \oplus U_{s}$ for $1 \leq s \leq 6$. Furthermore, let

$$
W_{1}=\left\langle y, y_{1}, x_{2}, \ldots, x_{k-1}\right\rangle, \quad W_{2}=\left\langle y, x_{1}, y_{2}, \ldots, y_{k-1}\right\rangle .
$$

Then all of $V_{1}, \ldots, V_{6}, W_{1}, W_{2}$ are totally singular $k$-dimensional subspaces with pairwise odd-dimensional intersections, so they are in the same $G$-orbit $X$. Adding $V_{1}, \ldots, V_{6}, W_{1}$, $W_{2}$ to $\mathcal{B}$ we see that any $g \in G_{(\mathcal{B})}$ fixes the subspaces $\langle x\rangle=V_{1} \cap V_{2},\langle y\rangle=W_{1} \cap W_{2}$ and $U=\left(V_{1}+V_{2}\right) \cap\left(W_{1}+W_{2}\right)$. Furthermore, $g \in G_{(\mathcal{B})}$ also fixes $U_{s}=V_{s} \cap U$ for each $s$, so $g_{U}$ is a scalar transformation by the definition of the $U_{s}$. Adding also the subspace

$$
W_{3}=\left\langle x+x_{1}, y-y_{1}, x_{2}, \ldots, x_{k-1}\right\rangle \in X
$$

to $\mathcal{B}$, we get that any $g \in G_{(\mathcal{B})}$ is a scalar transformation on the whole of $V$. Hence $b_{X}(G) \leq 9$ in this case.

Remark 3.4. By a more detailed argument, Burness, Guralnick and Saxl were able to calculate the exact base size for a classical group over an algebraically closed field acting on an orbit of subspaces of its natural module [9, Section 4]. While part of their constructions could be translated to the finite case, we had to give new constructions for other cases. (This is especially true for the orthogonal case, since, in contrast to the finite case discussed above, in any dimension there is just one type of non-degenerate orthogonal space over an algebraically closed field.)

3.2. Action on pairs of subspaces. In this subsection we handle the subspace actions arising from case (2) in the list after the statement of Theorem 3.1 .

Proposition 3.5. Let $G=P S L(V)=P S L_{d}(q)$, and let $M$ be the stabilizer in $G$ of a pair $U, W$ of nonzero subspaces, where $\operatorname{dim} U=k<d / 2, \operatorname{dim} W=d-k$, and either $U \subseteq W$ or $V=U \oplus W$. Let $X$ be the coset space $G / M$. Then

$$
b_{X}(G) \leq \frac{d}{k}+11 \leq 2 \frac{\log |G|}{\log |X|}+12 .
$$


Proof. Let $X_{k}$ be the set of all $k$-dimensional subspaces of $V$. A straightforward computation shows that $|X|<\left|X_{k}\right|^{2}$. Clearly $b_{X}(G) \leq b_{X_{k}}(G)$. Now the result follows from Theorem 3.3 and Proposition 3.2 .

3.3. Proof of Theorem 3.1. Let $G$ be an almost simple group with socle $G_{0}$, a classical group on $V$, a vector space of dimension $d$ over $\mathbb{F}_{q}$. Suppose $G$ acts faithfully and primitively on a set $\Omega$.

If the action of $G$ on $\Omega$ is not a subspace action, then $b(G) \leq 5$ by [7]. Hence we may assume that the action is a subspace action, so that one of the cases (1), (2), (3) listed after the statement of Theorem 3.1 holds.

In case (1), $\Omega=U^{G}$ is an orbit of $G$ on $k$-dimensional subspaces, for some $k$, and we can assume that $k \leq d / 2$ (by replacing $U$ with $U^{\perp}$ if necessary, in the case where $G_{0} \neq P S L(V)$, and by considering the equivalent action of $G$ on $(d-k)$-spaces, when $G_{0}=P S L(V)$ ). Now Theorem 3.3 and Proposition 3.2 give

$$
b\left(G_{0}\right) \leq \frac{d}{k}+11 \leq 2 \frac{\log |G|}{\log |\Omega|}+13 .
$$

Hence we can choose a set $\mathcal{B}$ of at most $\frac{d}{k}+11$ points of $\Omega$ such that $G_{(\mathcal{B})} \cap G_{0}=1$, so that $G_{(\mathcal{B})}$ is isomorphic to a subgroup of $G / G_{0}$. This is a soluble group possessing a normal series of length at most 3 with cyclic factor groups. Since the base size of a cyclic linear group is 1 , by [33], it follows that $b(G) \leq 2 \frac{\log |G|}{\log |\Omega|}+16$, as required.

Now consider case (2): here $G_{0}=P S L(V)$ and $\Omega=\{U, W\}^{G}$ where $U, W$ are subspaces of dimensions $k, d-k$ and either $U \subseteq W$ or $V=U \oplus W$. In the latter case, if $k=d / 2$ then $G_{0}$ has an element interchanging $U$ and $W$, and $(G, \Omega)$ is not a subspace action (it is a $\mathcal{C}_{2}$-action in the terminology of [7]). Hence we may assume that $k<d / 2$. Now Proposition 3.5 implies that $b\left(G_{0}\right) \leq 2 \frac{\log |G|}{\log |\Omega|}+12$, and this yields the result as above.

Finally, consider case (3): here $G_{0}=S p_{2 m}(q), p=2$ and $M \cap G_{0}=O_{2 m}^{ \pm}(q)$, where $M$ is a point-stabilizer in $G$. Regarding $G_{0}$ as the isomorphic orthogonal group $O_{2 m+1}(q)$, the set $\Omega$ is an orbit of $G_{0}$ on hyperplanes of the natural module $V_{2 m+1}(q)$. Hence $b_{\Omega}\left(G_{0}\right) \leq 2 m+1$, which is less than $2 \frac{\log |G|}{\log |\Omega|}+3$, and the conclusion follows again.

This completes the proof of Theorem 3.1.

\section{NON-AFFine PRIMITIVE PERMUtATION GROUPS}

In this section we prove the main Theorem 1.1 for primitive groups which are not of affine type.

Theorem 4.1. Let $G$ be a primitive permutation group of degree $n$. Assume that $G$ is not of affine type. Then $b(G) \leq 2(\log |G| / \log n)+24$.

According to the O'Nan-Scott theorem (see for example [24]), non-affine primitive groups are of the following types: almost simple, diagonal type, product type, and twisted wreath type. We shall deal with these types separately in the following subsections.

4.1. Almost simple groups. For this case we prove

Theorem 4.2. Let $G$ be an almost simple primitive permutation group of degree $n$. Then $b(G) \leq 2(\log |G| / \log n)+16$.

Proof. Theorems 2.1 and 3.1 give the result when the socle of $G$ is an alternating or classical group. For the remaining cases, the socle of $G$ is a group of exceptional Lie type or a sporadic group. In these cases we have $b(G) \leq 7$ by [10] and [11]. 
4.2. Diagonal type groups. Work of Fawcett [14] (and also Gluck, Seress, Shalev [17, Remark 4.3]) implies that, in the diagonal type case, we have

$$
b(G) \leq(\log |G| / \log n)+3 .
$$

4.3. Product type groups. Bases for primitive groups of product type were studied by Burness and Seress in [12]. We will use their notation. Let $\Omega=\Gamma^{k}$ for some set $\Gamma$ and integer $k \geq 2$. There exists a primitive group $H \leq \operatorname{Sym}(\Gamma)$ of almost simple type or of diagonal type such that the following holds. Let the socle of $H$ be $T$. Let $P$ be the (transitive) action of $G$ on the set of the $k$ direct factors of $\operatorname{Soc}(G)=T^{k}$. We have $T^{k} \leq G \leq H \curlywedge P$.

We recall two definitions. A distinguishing partition for a finite group $X$ acting on a finite set $\Sigma$ is a coloring of the points of $\Sigma$ in such a way that every element of $X$ fixing this coloring is contained in the kernel of the action of $X$ on $\Sigma$. The minimal number of parts (or colors) of a distinguishing partition is called the distinguishing number of $X$ and is denoted by $d(X)$.

Let $d(P)$ be the distinguishing number of the transitive permutation group $P$. By [13, Theorem 1.2], we have $d(P) \leq 48 \sqrt[k]{|P|}$.

4.3.1. The case when $H$ is almost simple. Assume that $H \leq \operatorname{Sym}(\Gamma)$ is an almost simple group with socle $T$. We follow not only [12] here but [13, §4]. However we avoid the use of the bound $|\operatorname{Out}(T)| \leq|T|^{\alpha}$, since this is expensive. Instead we use the estimate $|\operatorname{Out}(T)| \leq|\Gamma|$ found in [1, Lemma 2.7]. Thus $|G| \geq|T|^{k}|P| \geq\left(|H|^{k}|P|\right) /|\Gamma|^{k}$. This gives $\log \left(|H|^{k}|P|\right) / \log |\Omega| \leq(\log |G| / \log |\Omega|)+1$.

By using the idea of [12, Lemma 3.8] combined with Lemma 2.1 of [13], we see that

$$
b(G)<\frac{\log d(P)}{\log |\Gamma|}+1+b(H)<\frac{\log |P|}{\log |\Omega|}+b(H)+4,
$$

since $|\Gamma| \geq 5$. By Theorem 4.2, this gives

$$
b(G)<\frac{\log |P|}{\log |\Omega|}+2 \frac{\log |H|}{\log |\Gamma|}+20<2 \frac{\log \left(|H|^{k}|P|\right)}{\log |\Omega|}+20 \leq 2 \frac{\log |G|}{\log |\Omega|}+22 .
$$

4.3.2. The case when $H$ is of diagonal type. Now assume that $H$ is of diagonal type. Here $\operatorname{Soc}(H)=T=S^{\ell}$, where $S$ is a non-abelian simple group and $\ell \geq 2$. We have $S^{\ell} \leq H \leq S^{\ell}$. $(\operatorname{Out}(S) \times Q)$ where $Q \leq \operatorname{Sym}(\ell)$ is the permutation group induced by the conjugation action of $H$ on the $\ell$ factors of $S^{\ell}$.

The set $\Gamma$ can be thought of as the set of right cosets in $H$ of the subgroup $H_{0}=$ $(D \times Q) \cap H$ where $D$ denotes the diagonal subgroup of $\operatorname{Aut}(S)^{\ell}$. In particular, $|\Gamma|=|S|^{\ell-1}$. By (1), $b(H) \leq(\log |H| / \log |\Gamma|)+3 \leq 8$, provided that $\ell \leq|S|$. Thus, in view of (2), we may assume that $\ell \geq 3$.

Let $\mathcal{C}$ be the set of complete representatives of the right cosets in $H$ of the subgroup $H_{0}$ consisting of the elements of $S^{\ell}$ where the first coordinate is 1 . Let $s_{1}$ and $s_{2}$ be elements of $S$ such that they together generate $S$. Let $\gamma_{0}, \gamma_{1}, \gamma_{2} \in \mathcal{C}$ be those elements for which every coordinate of $\gamma_{0}$ is 1 , all but the first coordinate of $\gamma_{1}$ is $s_{1}$, and all but the first coordinate of $\gamma_{2}$ is $s_{2}$. Consider the pointwise stabilizer $Q_{0}$ of $\left\{H_{0} \gamma_{0}, H_{0} \gamma_{1}, H_{0} \gamma_{2}\right\}$. This group $Q_{0}$ is contained in the stabilizer $H_{0}$ of $H_{0} \gamma_{0}$. For any element $h_{0} \in H_{0}$ and any index $i$ in $\{1,2\}$, we have $H_{0} \gamma_{i} h_{0}=H_{0} \gamma$ for some $\gamma \in \mathcal{C}$ with 1 or $\ell-1>1$ entries equal to 1 . Moreover if $h_{0}$ is in $Q_{0}$, then the first case must hold. Since the only automorphism of $S$ fixing both $s_{1}$ and $s_{2}$ is the identity, we see that $Q_{0}$ is a subgroup of $Q$ leaving $\mathcal{C}$ invariant. Therefore, whenever $q_{0} \in Q_{0}$ and $\gamma \in \mathcal{C}$, we have $\left(H_{0} \gamma\right) q_{0}=H_{0} \gamma^{q_{0}}$ for $\gamma^{q_{0}} \in \mathcal{C}$. 
Let $\omega_{0}, \omega_{1}, \omega_{2}$ be those elements of $\Omega$ for which all $k$ coordinates of $\Omega$ are $H_{0} \gamma_{0}, H_{0} \gamma_{1}$, $H_{0} \gamma_{2}$, respectively. By the previous paragraph and the fact that $G \leq H \imath P$, the pointwise stabilizer in $G$ of $\left\{\omega_{0}, \omega_{1}, \omega_{2}\right\}$ is a permutation group $R$ permuting the $k \ell$ coordinates of the vectors in $S^{k \ell}$. More precisely, if the coordinates are labelled by the integers $1, \ldots, k \ell$, then $R$ is a permutation group on $\{1, \ldots, k \ell\}$ such that $\{j \ell+1 \mid j \in\{0, \ldots, k-1\}\}$ is $R$-invariant. Since $R$ is a subgroup of a transitive group on $k \ell$ points which has order at most $|G|$, we see, by [13, Theorem 1.2], that $d(R) \leq 48 \sqrt[k \ell]{|G|}$.

Consider a distinguishing partition $\mathcal{P}$ with $d(R)$ colors for the action of $R$ on $\{1, \ldots, k \ell\}$. Define a new coloring of the $R$-invariant subset $\{1, \ldots, k \ell\} \backslash\{j \ell+1 \mid j \in\{0, \ldots, k-1\}\}$ using no more than $d(R)^{2}$ colors in the following way. For any integers $j$ and $u$ with $0 \leq j \leq k-1$ and $1<u \leq \ell$ color $j \ell+u$ with the color $(\alpha, \beta)$ where $\alpha$ is the color of $j \ell+1$ in $\mathcal{P}$ and $\beta$ is the color of $j \ell+u$ in $\mathcal{P}$. Clearly, no non-identity element of $R$ preserves this new coloring. For $\ell \geq 3$, we see, by Lemma 2.1 of [13], that $G$ has a base $B$ containing $\left\{\omega_{0}, \omega_{1}, \omega_{2}\right\}$ such that

$$
b(G) \leq|B|=2 \frac{\log d(R)}{\log |S|}+4<2 \frac{\log |G|}{k \ell \log |S|}+6=2 \frac{\log |G|}{\log n}+6 .
$$

4.4. Twisted wreath product type groups. This type was treated in Burness and Seress [12, Section 4]. We follow their discussion. By the previous section we know that if $L$ is a primitive permutation group of product type acting on a set $\Omega$, then we have $b(L) \leq 2(\log |L| / \log |\Omega|)+22$. Let $G$ be a primitive permutation group of twisted wreath product type acting on the set $\Omega$. Then $G$ contains a regular normal subgroup $T^{k}$ isomorphic to the direct product of $k$ copies of a non-abelian simple group $T$. We may write $G=T^{k} P$ where $P$ is a transitive permutation group acting on $k$ points. As explained in [29, Section 3.6], we may embed $G$ in a group of product type $L$ which is of the form $\left(T^{2}\right)^{k} . P$. Thus $b(G) \leq b(L) \leq 2(\log |L| / \log |\Omega|)+22=2(\log |G| / \log |\Omega|)+24$.

This completes the proof of Theorem 4.1.

\section{Affine PRimitive Permutation Groups}

The main result of this section is

Theorem 5.1. Let $G$ be an affine primitive permutation group of degree $n$. Then $b(G) \leq$ $2(\log |G| / \log n)+16$.

Let $G$ be an affine primitive permutation group of degree $n$ with a point-stabilizer $H$. Then $G=V H \leq A G L(V)$, where $V$ is a finite vector space of order $n=p^{k}(p$ prime), and the stabilizer $H$ of the zero vector is an irreducible subgroup of $G L(V)$. Since $b(G)=b(H)+1$, Theorem 5.1 follows immediately from

Theorem 5.2. Let $H$ be a subgroup of $G L(V)$ acting irreducibly on the finite vector space $V$. Then $b_{V}(H) \leq 2(\log |H| / \log |V|)+17$.

In the above theorems, the multiplicative constant 2 is best possible, as shown by the following example (which completes the proof of Proposition 1.2).

Proposition 5.3. Let $V$ be a d-dimensional (d even) non-degenerate symplectic space over the finite field $\mathbb{F}_{q}$ and let $H=S p(V)$ with its natural action on $V$.

(i) Then $b_{V}(H)=d$.

(ii) If $G=V H \leq A G L(V)$ is the corresponding affine primitive permutation group, then for sufficiently large values of $q$, we have

$$
b(G)=\lfloor 2(\log |G| / \log n)\rceil-2 .
$$


Proof. (i) Clearly, any basis of $V$ (as a vector space) is also a base for $H$, so $b_{V}(H) \leq d$. For the equality, let $\left\{b_{1}, \ldots, b_{l}\right\} \in V$ be any set of vectors with $l \leq d-1$. Then there is a subspace $U \leq V$ containing $\left\{b_{1}, \ldots, b_{l}\right\}$ with $\operatorname{dim} U=d-1$. Hence it is enough to show that for every such subspace $U$, there exists a non-identity $g \in H$ that acts trivially on $U$.

Let $U \leq V$ be a subspace of dimension $d-1$ and let [, ] denote the non-degenerate symplectic bilinear form on $V$ preserved by $H$. Then the restriction of [, ] to $U$ is degenerate: there exists $0 \neq x \in U$ such that $\langle x\rangle=U^{\perp}$. Let $y \in V \backslash U$ be arbitrary. We claim that the map

$$
A: c y+u \mapsto c(y+x)+u\left(c \in \mathbb{F}_{q}, u \in U\right)
$$

is an element of $H$, which acts trivially on $U$. To see this, let $c, d \in \mathbb{F}_{q}$ and $u, v \in U$. Then we have $[A(c y+u), A(d y+v)]=[c y+u+c x, d y+v+d x]=[c y+u, d y+v]$, proving the claim.

(ii) This follows from a simple computation using the order formula for $|S p(V)|$.

It remains to prove Theorem 5.2. We do this in the following two subsections.

5.1. Primitive linear groups. In this subsection we prove Theorem 5.2 in the case where $H \leq G L(V)$ acts primitively on $V$ as a linear group. In fact we prove the following stronger bound for this case.

Theorem 5.4. Let $V$ be a finite vector space, and let $H \leq G L(V)$ be an irreducible, primitive linear group on $V$. Then one of the following holds:

(i) $b(H) \leq 15$;

(ii) $b(H) \leq 2 \frac{\log |H|}{\log |V|}+9$.

A version of Theorem 5.4 was proved in [26, 27] with a much worse multiplicative constant, and unspecified constants in place of the constants 15 and 9 . The proof of Theorem 5.4 will be along the lines of that proof. However, in order to make our constants explicit (and small), we need to improve several of the results in [26, 27].

For a field $\mathbb{F}_{q}$ and a positive integer $m$, by the natural module for the symmetric or alternating group $\operatorname{Sym}(m)$ or $\operatorname{Alt}(m)$ over $\mathbb{F}_{q}$, we mean the fully deleted permutation module of dimension $m^{\prime}=m-\delta$, where $\delta \in\{1,2\}$.

The first result is a version of Proposition 2.2 of [26] with an explicit constant:

Proposition 5.5. Let $V=V_{d}(q)\left(q=p^{e}\right)$ and $G \leq G L(V)$, and suppose that $E(G)$ is quasisimple and absolutely irreducible on $V$. Then one of the following holds:

(i) $E(G)=\operatorname{Alt}(m)$ and $V$ is the natural $\operatorname{Alt}(m)$-module over $\mathbb{F}_{q}$, of dimension $d=$ $m-\delta(\delta \in\{1,2\})$;

(ii) $E(G)=C l_{d}\left(q_{0}\right)$, a classical group with natural module of dimension d over a subfield $\mathbb{F}_{q_{0}}$ of $\mathbb{F}_{q}$;

(iii) $b(G) \leq 6$.

Proof. This is proved in $[22$.

The next result is an explicit version of [26, Proposition 3.6].

Proposition 5.6. Let $V=V_{d}(q)\left(q=p^{e}\right)$ and $G \leq G L(V)$, and suppose that the Fitting subgroup $F(G)$ is absolutely irreducible on $V$. Then $b(G) \leq 13$.

Proof. We begin by arguing exactly as in the proof of [26, 3.6] that $F=F(G)$ can be taken to be the central product of an extraspecial group $s^{1+2 m}$ and the group $Z=\mathbb{F}_{q}^{*}$ of scalars, where $s$ is a prime and $d=s^{m}$. We can also assume that $G=N_{G L(V)}(F)$, so that 
$G / F$ is isomorphic to either $S p_{2 m}(s)$ or $O_{2 m}^{ \pm}(2)$, with $s=2$ in the latter case. Moreover, $q \equiv 1 \bmod s$, and also $q \equiv 1 \bmod 4$ if $s=2$ and $G / F \cong S p_{2 m}(2)$.

Define $F^{0}=F \cdot Z(G / F)$, an extension of $F$ by a group of order at most 2. By [32], there are three vectors $v_{1}, v_{2}, v_{3} \in V$ such that $F_{v_{1} v_{2} v_{3}}^{0}=1$. So if we let $J=G_{v_{1} v_{2} v_{3}}$, then $J \cap F^{0}=1$ and $J \cong J F^{0} / F^{0}$ is isomorphic to a subgroup of $P S p_{2 m}(s)$ or $O_{2 m}^{ \pm}(2)$.

Obviously $b(G) \leq 3+b(J)$. Assume for a contradiction that

$$
b(J)>10 .
$$

Then clearly $d=\operatorname{dim} V>10$, and $V^{10}=\bigcup_{h \in J \backslash 1} C_{V^{10}}(h)$. Hence

$$
|V|^{10} \leq \sum_{h \in J \backslash 1}\left|C_{V}(h)\right|^{10} .
$$

For $h \in J \backslash 1$, Theorem 4.1 of [19] shows that there are $2 m+1$ conjugates of $h$ that generate $G$ modulo $F$, and hence there are $2 m+2$ conjugates of $h$ generating $G$. It follows that

$$
\operatorname{dim} C_{V}(h) \leq\left(1-\frac{1}{2 m+2}\right) \operatorname{dim} V .
$$

Hence (3) gives $|V|^{10 /(2 m+2)} \leq|J|$, and so as $|V|=q^{d}=q^{s^{m}}$, we have

$$
q^{10 s^{m} /(2 m+2)} \leq|J| \leq\left\{\begin{array}{l}
\left|P S p_{2 m}(s)\right|, s \text { odd }, q \equiv 1 \bmod s \\
\left|S p_{2 m}(2)\right|, s=2, q \equiv 1 \bmod 4 \\
\left|O_{2 m}^{ \pm}(2)\right|, s=2, q \text { odd } .
\end{array}\right.
$$

Straightforward computation shows that the only possible values satisfying (4) are $s=2$, $q=3$ and $m=4$ or 5 . For $m=5$ we have $G / F \cong O_{10}^{ \pm}(2)$, and in the above argument, [19, 4.1] shows that we may replace $2 m+2$ by $2 m+1$ in (44), yielding a contradiction. And if $m=4$ then $d=2^{4}=16$ and it is very easy to argue directly that $b(G) \leq 13$, as required.

The next result is an improvement of Lemma 3.7 of [26].

Proposition 5.7. (i) Let $\mathbb{F}_{q_{0}}$ be a subfield of $\mathbb{F}_{q}$, let $q=q_{0}^{r}$, and let $M=\mathbb{F}_{q}^{*} G L_{d}\left(q_{0}\right) \leq$ $G L_{d}(q)=G L(V)$, where $\mathbb{F}_{q}^{*}$ denotes the group of scalars. Then for the action of $M$ on $V$ we have

$$
b(M) \leq \frac{d}{r}+2 .
$$

(ii) Let $q=p^{r}$ with $p$ prime, and let $M=\mathbb{F}_{q}^{*} \operatorname{Sym}(m) \leq G L_{m^{\prime}}(q)=G L(V)$, where $V$ is the natural module for $\operatorname{Sym}(m)$ over $\mathbb{F}_{q}$, of dimension $m^{\prime}=m-\delta(\delta \in\{1,2\})$. Then

$$
b(M) \leq \frac{\log _{p} m}{r}+4 .
$$

Proof. (i) Let $\lambda_{1}, \ldots, \lambda_{r}$ be an $\mathbb{F}_{q_{0}}$-basis for $\mathbb{F}_{q}$, and let $e_{1} \ldots, e_{d}$ be the standard basis for $V=\mathbb{F}_{q}^{d}$ (that is, $e_{i}=(0, \ldots, 1, \ldots 0)$ where the 1 is in the $i^{t h}$ coordinate). Write $d=k r+l$ with $k, l \in \mathbb{Z}$ and $0 \leq l<r$, and define

$$
\begin{aligned}
& v_{i}=\sum_{j=(i-1) r+1}^{i r} \lambda_{j} e_{j} \quad(1 \leq i \leq k), \\
& v_{k+1}=\sum_{j=k r+1}^{d} \lambda_{j} e_{j} .
\end{aligned}
$$

If we write $M_{0}=G L_{d}\left(q_{0}\right)$, it is easy to see that $\left(M_{0}\right)_{v_{1} \ldots v_{k+1}}=1$. Hence if $J=M_{v_{1} \ldots v_{k+1}}$ then $J \cong J M_{0} / M_{0}$ is cyclic, and so by [33, 3.1], $J$ has a base of size 1 . Thus $b(M) \stackrel{\leq}{\leq}$ $k+2 \leq \frac{d}{r}+2$.

(ii) This follows directly from the proof of [26, 3.7(ii)]. 
As in [26], for $H \leq G L(V)$ define $b^{*}(H)$ to be the minimal size of a set $B$ of vectors such that any element of $H$ that fixes every 1-space $\langle v\rangle$ with $v \in B$ is necessarily a scalar multiple of the identity. We call such a set $B$ a strong base for $H$. By [26, 3.1],

$$
b(H) \leq b^{*}(H) \leq b(H)+1 .
$$

Next we give an improvement of Lemma 3.3(iii) of [26].

Lemma 5.8. Let $V_{1}, V_{2}$ be vector spaces over $\mathbb{F}_{q}$ with $\operatorname{dim} V_{i}=n_{i}$ and $n_{1} \leq n_{2}$, and let $H_{i} \leq G L\left(V_{i}\right)$ for $i=1,2$. Denote by $H_{1} \otimes H_{2}$ the image of $H_{1} \times H_{2}$ acting in the natural way on the tensor product $V_{1} \otimes V_{2}$.

If $n_{1} \leq b^{*}\left(H_{2}\right)$, then

$$
b\left(H_{1} \otimes H_{2}\right) \leq \frac{b^{*}\left(H_{2}\right)}{n_{1}}+3 .
$$

Proof. We follows the proof of [26, Lemma 3.3(iii)]. Let $b=b^{*}\left(H_{2}\right)$. Assume $n_{1} \leq b$, and let $y_{1}, \ldots, y_{b}$ be a linearly independent strong base for $H_{2}$ in $V_{2}$. Let $x_{1}, \ldots, x_{n_{1}}$ be a basis of $V_{1}$.

Write $b=r n_{1}+s$ with $r, s$ integers and $0 \leq s<n_{1}$ For $1 \leq i \leq r$ define

$$
v_{i}=\sum_{k=1}^{n_{1}} x_{k} \otimes y_{(i-1) n_{1}+k}, \quad W_{i}=\left\langle x_{k} \otimes y_{(i-1) n_{1}+k}: 1 \leq k \leq n_{1}\right\rangle,
$$

and set $v_{r+1}=\sum_{k=1}^{s} x_{k} \otimes y_{r n_{1}+k}, W_{r+1}=\left\langle x_{k} \otimes y_{r n_{1}+k}: 1 \leq k \leq s\right\rangle$.

Consider the stabilizer $L=\left(H_{1} \otimes H_{2}\right)_{v_{1} \ldots v_{r+1}}$. By Lemma 3.3(i) of [26], $L$ stabilizes $V_{1} \otimes W_{i}$ for all $1 \leq i \leq r+1$.

Next choose $C, D \in S L_{n_{1}}(q)$ generating $S L_{n_{1}}(q)$, and for each $i$, define $\gamma_{i}=C \otimes 1, \delta_{i}=$ $D \otimes 1 \in G L\left(V_{1} \otimes W_{i}\right)$. Let

$$
v=\sum_{i=1}^{r+1} v_{i} \gamma_{i}, w=\sum_{i=1}^{r+1} v_{i} \delta_{i} .
$$

At this point the argument at the end of the proof of [26, 3.3(iii)] shows that $L_{v w}=1$. Hence $b\left(H_{1} \otimes H_{2}\right) \leq r+3 \leq \frac{n_{1}}{b}+3$, as required.

The next result is Theorem 1 of [27], with an explicit constant $C=14$. The proof is identical to that in [27, but using Propositions 5.5 and 5.6 at the end to justify that $C=14$ works.

Proposition 5.9. Let $V=V_{d}(q)$, and let $H$ be a subgroup of $\Gamma L(V)$ such that $H$ acts primitively on $V$ and $H^{0}:=H \cap G L(V)$ is absolutely irreducible on $V$. Suppose that $b^{*}\left(H^{0}\right)>14$. Then

$$
H^{0} \leq H_{0} \otimes \bigotimes_{i=1}^{s} \operatorname{Sym}\left(m_{i}\right) \otimes \bigotimes_{i=1}^{t} \mathrm{Cl}_{d_{i}}\left(q_{i}\right),
$$

where $s+t \geq 1$ and the following hold:

(i) $H_{0} \leq G L_{d_{0}}(q)$ with $b^{*}\left(H_{0}\right) \leq 14$

(ii) each factor $\operatorname{Sym}\left(m_{i}\right)<G L_{m_{i}^{\prime}}(q)$ and $\mathrm{Cl}_{d_{i}}\left(q_{i}\right) \leq G L_{d_{i}}(q)$ is acting on the natural module over $\mathbb{F}_{q}$, where $m_{i}^{\prime}=m_{i}-\delta_{i}, \delta_{i} \in\{1,2\}$

(iii) $d=d_{0} \cdot \prod_{1}^{s} m_{i}^{\prime} \cdot \prod_{1}^{t} d_{i}$

(iv) $F^{*}\left(H^{0}\right)$ contains $\prod_{1}^{s} \operatorname{Alt}\left(m_{i}\right) \cdot \prod_{1}^{t} \mathrm{Cl}_{d_{i}}\left(q_{i}\right)^{(\infty)}$.

The next result is an improvement of [27, Proposition 2].

Proposition 5.10. Let $H, H^{0}$ be as in Proposition 5.9, with $b^{*}\left(H^{0}\right)>14$. Take $m_{s}^{\prime}=$ $\max \left(m_{i}^{\prime}: 1 \leq i \leq s\right)$ and $d_{t}=\max \left(d_{i}: 1 \leq i \leq t\right)$ (define these to be 0 if $s=0$ or $t=0$, respectively). 
(i) Suppose $t \geq 1$ and $m_{s}^{\prime} \leq d_{t}$, and let $q=q_{t}^{r}$. Then $d<d_{t}^{2}$, $d_{t} \geq 14$, and

$$
b\left(H^{0}\right) \leq b\left(G L_{d / d_{t}}(q) \otimes G L_{d_{t}}\left(q_{t}\right)\right) \leq \frac{d_{t}^{2}}{d r}+5 .
$$

(ii) Suppose $s \geq 1$ and $m_{s}^{\prime}>d_{t}$, and let $q=p^{r}$. Then $d<\left(m_{s}^{\prime}\right)^{2}, m_{s}^{\prime} \geq 14$, and

$$
b\left(H^{0}\right) \leq b\left(G L_{d / m_{s}^{\prime}}(q) \otimes \operatorname{Sym}\left(m_{s}\right)\right) \leq \frac{m_{s} \log _{p} m_{s}}{d r}+6 .
$$

Proof. We follow the proof of [27, Proposition 2], but as the constants are different we give a few details.

We proceed by induction on $s+t$. For the base case $s+t=1$, we have $H^{0} \leq H_{0} \otimes M$, where $M=\mathrm{Cl}_{d_{1}}\left(q_{1}\right)$ or $\operatorname{Sym}\left(m_{1}\right)$. Consider the first case, and write $q=q_{1}^{r}$. Proposition 5.7 gives $b(M) \leq \frac{d_{1}}{r}+2$, hence also $b^{*}(M) \leq \frac{d_{1}}{r}+3$. If $d_{0}=1$ the conclusion in (i) is immediate, so assume $d_{0} \geq 2$. As in the proof of [27, Proposition 2], we see that $d_{0} \leq d_{1}$. Then Lemma 5.8 gives

$$
\begin{aligned}
b\left(H^{0}\right) & \leq \frac{b^{*}(M)}{d_{0}}+3 \\
& \leq \frac{d_{1}}{r d_{0}}+\frac{3}{d_{0}}+3 \\
& <\frac{d_{1}}{r d_{0}}+5,
\end{aligned}
$$

so that (i) holds (note that $d_{1} \geq 14$ by [26, 3.3(ii)]). Similarly (ii) holds when $M=$ $\operatorname{Sym}\left(m_{1}\right)$.

Now assume $s+t \geq 2$. Let $m$ be the maximum of $d_{t}$ and $m_{s}^{\prime}$, and write $M$ for the corresponding group $\mathrm{Cl}_{d_{t}}\left(q_{t}\right)$ or $\operatorname{Sym}\left(m_{s}\right)$. Note that $m \geq 14$, since otherwise [26, 3.3(ii)] implies that $b^{*}\left(H^{0}\right) \leq 14$. Let $N$ be the tensor product of $H_{0}$ and the other factors $\mathrm{Cl}_{d_{i}}\left(q_{i}\right), \operatorname{Sym}\left(m_{i}\right)$, so that $H^{0} \leq N \otimes M$. If $b^{*}(N) \leq 14$ the conclusion follows as in the $s+t=1$ case, so assume $b^{*}(N)>14$.

Let $m^{\prime}$ be the largest among the dimensions $d_{i}, m_{i}^{\prime}$ omitting $m$, and write $N_{1}$ for the corresponding group $\mathrm{Cl}_{d_{i}}\left(q_{i}\right)$ or $\operatorname{Sym}\left(m_{i}\right)$.

Consider the case where $N_{1}=\mathrm{Cl}_{d_{i}}\left(q_{i}\right)$. Let $q=q_{i}^{u}$. By induction we have

$$
b^{*}(N) \leq b(N)+1 \leq \frac{d_{i}^{2} m}{d u}+6 \leq \frac{d_{i}}{u}+6 .
$$

Suppose $d \geq m^{2}$. Then $b^{*}(N) \geq m$ by [26, 3.3(iv)], so Lemma 5.8 implies that

$$
b\left(H^{0}\right) \leq \frac{b^{*}(N)}{m}+3 \leq \frac{d_{i}}{u m}+\frac{6}{m}+3 .
$$

Since $m \geq d_{i}$ and $m>14$, this yields $b\left(H^{0}\right)<5$, a contradiction. Hence $d<m^{2}$ in this case. Now the conclusion of the proposition follows by the argument given for the $s+t=1$ case.

Finally, consider the case where $N_{1}=\operatorname{Sym}\left(m_{i}\right)$. Let $q=p^{r}$. By induction,

$$
b^{*}(N) \leq b(N)+1 \leq \frac{\left(m_{i} \log _{p} m_{i}\right) \cdot m}{d r}+7 \leq \frac{\log _{p} m_{i}}{r}+8 .
$$

Now the argument of the previous paragraph gives the conclusion.

\section{Proof of Theorem 5.4}

We are now in a position to prove Theorem 5.4 .

We begin just as in the proof of [27, Corollary 3]. Suppose $H \leq G L(V)$ acts primitively and irreducibly on a finite vector space $V$ defined over a field of size $q_{0}$. Choose $q=q_{0}^{r}$ maximal such that $H \leq \Gamma L_{d}(q) \leq G L_{d r}\left(q_{0}\right)$. Write $H^{0}=H \cap G L_{d}(q)$ and $V=V_{d}(q)$. By [16, 12.1], $H^{0}$ is absolutely irreducible on $V$. 
If $b^{*}\left(H^{0}\right) \leq 14$ then $b(H) \leq 15$, and the conclusion of Theorem 5.4 holds. So assume now that $b^{*}\left(H^{0}\right)>14$. Then $H^{0}$ is given by Proposition 5.9, and (i) or (ii) of Proposition 5.10 holds.

Consider case (i) of Proposition [5.10. Write $m=d_{t}$ and $q=q_{t}^{r}$. Then $d<m^{2}$, $H^{0} \triangleleft C l_{m}\left(q_{t}\right), m \geq 14$, and

$$
b\left(H^{0}\right) \leq \frac{m^{2}}{d r}+5
$$

From the order formulae for classical groups, we see that

$$
\log _{q_{t}}\left(\left|H^{0}\right|\right) \geq \log _{q_{t}}\left|\Omega_{m}^{ \pm}\left(q_{t}\right)\right| \geq \frac{1}{2} m(m-1)-1 .
$$

Hence

and so (5) gives

$$
\frac{\log |H|}{\log |V|} \geq \frac{\frac{1}{2} m(m-1)-1}{r d}
$$

$$
b\left(H^{0}\right) \leq 2 \frac{\log |H|}{\log |V|}+5+\frac{m+2}{r d}<2 \frac{\log |H|}{\log |V|}+7 .
$$

This completes the proof in case (i) of Proposition 5.10.

To conclude, consider case (ii) of Proposition 5.10. Write $m=m_{s}, m^{\prime}=m_{s}^{\prime}$ and $q=p^{r}$. Then $d<m^{2}, H^{0} \triangleleft \operatorname{Alt}(m), m^{\prime} \geq 14$, and

$$
b\left(H^{0}\right) \leq \frac{m \log _{p} m}{d r}+6 .
$$

Now $\left|H^{0}\right| \geq \frac{1}{2} m !>\frac{1}{2}\left(\frac{m}{e}\right)^{m}$, and so

$$
\frac{\log |H|}{\log |V|} \geq \frac{m\left(\log _{p} m-\log _{p} e\right)-1}{r d} .
$$

Hence (6) gives

$$
b\left(H^{0}\right) \leq \frac{\log |H|}{\log |V|}+6+\frac{m \log _{2} e+3}{r d}<\frac{\log |H|}{\log |V|}+8,
$$

giving the conclusion of Theorem 5.4 (actually, a stronger version, with the constant 2 replaced by 1 ).

This completes the proof of Theorem 5.4.

5.2. Imprimitive linear groups. It remains to prove Theorem 5.2 in the case where the irreducible linear group $H \leq G L(V)$ acts imprimitively on $V$. Assume that $H$ preserves the direct sum decomposition $V=V_{1} \oplus \cdots \oplus V_{t}$ where $V_{1}, \ldots, V_{t}$ are subspaces of $V$, and $t>1$ is chosen maximally. Let $H_{1}$ be the stabilizer of $V_{1}$ in $H$. Let us denote the minimal base size of the action $K_{1}$ of $H_{1}$ on $V_{1}$ by $b\left(K_{1}\right)$. By the choice of $t$, the action of $K_{1}$ on $V_{1}$ is primitive, so by Theorem [5.4, we have $b\left(K_{1}\right) \leq 15$ or

$$
b\left(K_{1}\right) \leq 2\left(\log \left|K_{1}\right| / \log \left|V_{1}\right|\right)+9 .
$$

If $b\left(K_{1}\right) \leq 15$, then, by [13, Theorem 3.4] and its proof, we have

$$
b(H) \leq \log |H| / \log |V|+b\left(K_{1}\right)+1+\left(\log 48 / \log \left(2^{b\left(K_{1}\right)}\right)\right) \leq \log |H| / \log |V|+17 .
$$

So assume now that $b\left(K_{1}\right) \geq 16$ and $b\left(K_{1}\right) \leq 2\left(\log \left|K_{1}\right| / \log \left|V_{1}\right|\right)+9$. In that case our proof strictly follows the arguments of [13, but in order to be able to prove Theorem 5.2 we need to give more precise estimates for the constants appearing there. In the proof we will freely use the concepts and notation of [13].

A main step in proving [13, Theorem 3.17] was to reduce the problem for linear groups which do not preserve any tensor product decomposition of the vector space (possibly over 
a proper field extension of the base field). In order for the reduction argument to work, a generalisation of the problem was needed.

Let us view $H$ not just as a subgroup of $G L(V)$ but also as an abstract group. We will define certain maps $X: H \rightarrow G L(V)$. For this let $T_{V}$ denote the group

$$
T_{V}=\left\{g \in G L(V) \mid g\left(V_{i}\right)=V_{i} \text { and }\left.g\right|_{V_{i}} \in Z\left(G L\left(V_{i}\right)\right) \quad \forall 1 \leq i \leq t\right\} \simeq\left(\mathbb{F}_{q}^{\times}\right)^{t}
$$

where $\mathbb{F}_{q}$ is the field of definition for $V$. According to [13, Definition 3.5] we say that a map $X: H \rightarrow G L(V)$ is a $\left(\bmod T_{V}\right)$-representation of $H$ if the following two properties hold: (1) $X(g)$ normalizes $T_{V}$ for every $g \in H$; and (2) $X(g h) T_{V}=X(g) X(h) T_{V}$ for every $g, h \in H$. We will always consider $\left(\bmod T_{V}\right)$-representations $X$ of $H$ such that the group $X(H) T_{V}$ acts transitively on the set of factors $\Pi=\left\{V_{1}, \ldots, V_{t}\right\}$ of the above direct sum decomposition of $V$. Let $b_{X}(H)$ denote the minimal base size of the group $X(H) T_{V}$.

In the rest of this subsection we will show that if $X$ is an imprimitive irreducible linear representation of $H$ preserving the decomposition $V=V_{1} \oplus \cdots \oplus V_{t}$, then $b_{X}(H) \leq$ $2(\log |H| / \log |V|)+17$, or $b(H) \leq 2(\log |H| / \log |V|)+17$. Theorem 5.2 will follow by specializing to the case when $X$ is the identity.

If the representation of $H$ is alternating-induced in the sense of [13, Section 3.2], then $b(H) \leq 2(\log |H| / \log |V|)+17$, by [13, Theorem 3.9].

By [13, Section 3.4] (and especially by [13, Corollary 3.15]), it is sufficient to establish the proposed bound in the claim for $b(H)$ or for $b_{X}(H)$ in case $X$ is a (not necessarily linear) $\left(\bmod T_{V}\right)$-representation of $H$ which is classical-induced satisfying the multiplicity-free condition, in the sense of [13, Section 3.3]. Let $X$ be such a representation of $H$.

Let $N$ be the kernel of the action of $H$ on $\Pi$. Let $\mathfrak{X}: H \rightarrow N_{G L(V(p))}\left(T_{V}\right) / T_{V}$ be the homomorphism defined by $\mathfrak{X}(h):=X(h) T_{V} / T_{V}$ where $V(p)$ denotes the vector space $V$ defined over the prime field (of size $p$ ) of $\mathbb{F}_{q}$ and where $h \in H$. In [13, Section 3.3] a bound is given for $b_{X}(H)$. By the argument after [13, Theorem 3.11] (from the second to the fifth paragraphs), we get $b_{X}(H)<(\log |H| / \log |V|)+12$ when $\mathfrak{X}(N)=1$. So assume that $\mathfrak{X}(N) \neq 1$.

We use the notation (and a minor modification of the argument) of the paragraph following [13, Theorem 3.11]. In this case $\operatorname{Soc}(\mathfrak{X}(N))$ is a subdirect product of isomorphic simple classical groups $S_{1}, \ldots, S_{t}$. The linking factor, denoted by $r$, is at most 2 . We have $|N| \geq\left|S_{1}\right|^{t / r}$. Since $b\left(K_{1}\right) \geq 16$, the center of $K_{1}$ has size less than $\left|V_{1}\right|^{1 / 16}$ and $\left|\operatorname{Out}\left(S_{1}\right)\right| \leq\left|V_{1}\right|^{6 / 16}$, (the latter by [18, Lemma 7.8]). Thus $\left|S_{1}\right| \geq\left|K_{1}\right| /\left|V_{1}\right|^{1 / 2}$, which implies $|N| \geq\left|K_{1}\right|^{t / r} /|V|^{1 / 2}$.

Assume that $r=1$. By Theorem [5.4, we have

$$
b\left(K_{1}\right) \leq 2\left(\log \left|K_{1}\right| / \log \left|V_{1}\right|\right)+9=2\left(\log \left(\left|K_{1}\right|^{t}\right) / \log |V|\right)+9 \leq 2(\log |N| / \log |V|)+10 .
$$

By [13, Theorem 3.4] and its proof, we then get $b(H) \leq 2(\log |H| / \log |V|)+12$ since $\log \left|V_{1}\right| \geq 16$.

Now assume that $r=2$, so $t=2 k$ for some integer $k$. By changing the order (if necessary) of the summands in the direct sum $V=\oplus_{i=1}^{t} V_{i}$, we can assume that the sets $\Delta_{i}:=\left\{V_{2 i-1}, V_{2 i}\right\}$, for all $i$ with $1 \leq i \leq k$, form a system of $H$-blocks with $S_{\Delta_{i}}:=$ $\operatorname{Soc}\left(\mathfrak{X}_{\Delta_{i}}(N)\right)$ a full diagonal subgroup of $S_{2 i-1} \times S_{2 i}$. Here $\mathfrak{X}_{\Delta_{i}}$ is defined as follows. Put $V_{\Delta_{i}}:=V_{2 i-1} \oplus V_{2 i}$ and let $T_{V_{\Delta_{i}}}$ be defined analogously as $T_{V}$. Let $X_{i}: N_{H}\left(\Delta_{i}\right) \rightarrow G L\left(V_{\Delta_{i}}\right)$ be the $\left(\bmod T_{V_{\Delta_{i}}}\right)$-representation of $N_{H}\left(\Delta_{i}\right)$ obtained naturally from $X$ by restricting first from $H$ to $N_{H}\left(\Delta_{i}\right)$ and then from $V$ to $V_{\Delta_{i}}$. Now define $\mathfrak{X}_{\Delta_{i}}$ to be the homomorphism given by $\mathfrak{X}_{\Delta_{i}}(h):=X_{i}(h) T_{V_{\Delta_{i}}} / T_{V_{\Delta_{i}}}$ for $h \in N_{H}\left(\Delta_{i}\right)$. The multiplicity-free condition (and the definition of $\Delta_{i}$ ) guarantees that there are no functions $\varphi_{i}: V_{2 i} \rightarrow V_{2 i-1}$ and 
$\lambda_{i}: N_{H}\left(\Delta_{i}\right) \rightarrow \mathbb{F}_{q}^{\times}$such that $\varphi_{i}$ is a semilinear invertible map and

$$
X_{2 i-1}(g)=\lambda_{i}(g) \cdot \varphi_{i} X_{2 i}(g) \varphi_{i}^{-1}
$$

for every $g \in C_{H}\left(\Delta_{i}\right)$. By using [21, Theorem 2.1.4], it follows that $S_{1} \simeq P S L\left(V_{1}\right)$. Thus, $|N| \geq\left(q^{\operatorname{dim}^{2} V_{1}-\operatorname{dim} V_{1}}\right)^{t / 2} /|V|^{1 / 2}$, which means that

$$
b_{X_{\Delta_{1}}}\left(N_{H}\left(\Delta_{1}\right)\right) \leq \operatorname{dim} V_{1} \leq 2(\log |N| / \log |V|)+2 .
$$

Now we can apply [13, Theorem 3.4] to the $H$-invariant direct sum decomposition $V=$ $\oplus_{i=1}^{k} V_{\Delta_{i}}$ to deduce that $b_{X}(H) \leq 2(\log |H| / \log |V|)+3+\log 48<2(\log |H| / \log |V|)+9$.

This completes the proof of Theorem 5.2 .

\section{Proof of Corollary 1.3}

In this section we will prove Corollary 1.3. Let $G$ be a primitive permutation group of degree $n$. For later use, we recall the definition of standard actions of almost simple primitive groups: these occur for groups with socle an alternating group $\operatorname{Alt}(m)$ or a classical group. In the former case they are actions on an orbit of subsets or partitions of $\{1, \ldots, m\}$; and in the latter, they are subspace actions (as defined in Section 3).

Assume first that $G$ is $\operatorname{Sym}(m)$ or $\operatorname{Alt}(m)$ for some integer $m \geq 5$. We consider standard actions of $G$.

If the action of $G$ is on a set of partitions of the underlying set of size $m$, then $b(G) \leq$ $\log n+4$ by Theorem 2.7. The right hand side is less than $\sqrt{n}$ for $n \geq 256$, and $b(G)<12$ otherwise.

Now assume that $G$ acts on $k$-element subsets of $\{1, \ldots, m\}$ for some integer $k$ with $2 \leq k \leq m / 2$ and $n=\left(\begin{array}{c}m \\ k\end{array}\right)$. Assume also that $b(G) \geq 26$.

Let $k^{2} \leq m$. Then, by Theorem 2.2, $b(G) \leq \frac{2 m}{k+1}+1$. Since $b(G) \geq 26$, we have $m \geq 38$ and $n \geq 625$. For $k=2$ and $n \geq 625$ we get $b(G) \leq \frac{2 m}{3}+1<\sqrt{n}$. For $k \geq 3$ and $n \geq 625$ we find that $m^{2} \leq\left(\begin{array}{c}m \\ k\end{array}\right)=n$ and so $b(G)<\sqrt{n}$.

Let $k^{2}>m$. Then $k \geq 3$ since $m \geq 5$. By Theorem [2.2, $b(G) \leq\left\lceil\log _{\lceil t\rceil}(m)\right\rceil(\lceil t\rceil-1)$ where $t=m / k$. Since $b(G) \geq 26$, this forces $m \geq 18$. In particular $k \geq 5$ and $n \geq 625$. Thus assume that $m \geq 18, k \geq 5$, and $n \geq 625$. Let $k \geq 8$. By Theorem 2.2 and the assumption $k^{2}>m$, we have $b(G) \leq(\log m+1) t<(2 \log k+1) t$. Since $t \geq 2$, it follows that $(2 \log k+1) t<t^{k / 2}=\left(\frac{m}{k}\right)^{k / 2}<\sqrt{\left(\begin{array}{c}m \\ k\end{array}\right)}$. If $5 \leq k \leq 7$, then $b(G) \leq 6(\log m+1)$, by Theorem 2.2 (recall that $m<k^{2}$ ). The right hand side is less than $\sqrt{\left(\begin{array}{c}m \\ k\end{array}\right)}$ provided that $m \geq 18$ and $5 \leq k \leq m / 2$.

Next assume that $G$ is a group as in Section 4.3.1, Let us use the notation and results of that section. By (2), we have $b(G)<\log k+1+n^{1 / k}$. For $k \geq 3$ this is less than $\sqrt{n}$ since $n \geq 5^{k} \geq 125$. Now let $k=2$. Then $G$ is a subgroup of $\operatorname{Sym}(t)$ ¿ $\operatorname{Sym}(2)$ with $n=t^{2}$. In this case $b(G) \leq t$, that is, $b(G) \leq \sqrt{n}$.

At this point, by [28, Theorem 1.1], we may assume that $|G| \leq n^{1+\log n}$ (and $n \geq 26$ ).

If $G$ is as in Section 4.3.2 or as in Section 4.4, then $n>2500$, and so by Theorem 1.1,

$$
b(G) \leq 2 \frac{\log |G|}{\log n}+24 \leq 2 \log n+26<\sqrt{n} .
$$

If $G$ is as in Section 4.2, we use Fawcett's [14] bound $b(G) \leq(\log |G| / \log n)+3$ to obtain $b(G) \leq \log n+4<\sqrt{n}$ provided $n \neq 60$. If $n=60$, then $|G| \leq 4 n^{2}$, and so $b(G) \leq 5$.

Assume that $G$ is almost simple. If the action of $G$ is non-standard, then $b(G) \leq 7$ by [7] and [10]. Thus assume that the action of $G$ is standard. In particular the socle of $G$ is 
a simple classical group or an alternating group. The case when the socle is an alternating group was treated before.

Let $G$ be an almost simple group with socle a classical group with natural module a vector space of dimension $d$ over some finite field. Since $|G| \leq n^{1+\log n}$ and $b(G) \leq$ $2(\log |G| / \log n)+16$, the latter by Theorem 3.1, we see that $b(G)<\sqrt{n}$ for $n \geq 1600$. Thus assume that $n<1600$. By Theorem 3.3 and Section 3.3 , we have $b(G) \leq d+14$. By [21, Table 5.2.A], we find that $d$ must be at most 11 , and so $b(G) \leq 25$.

Finally, assume that $G$ is of affine type with $n \geq 4$. Put $n=p^{d}$ where $p$ is a prime and $d$ is an integer. Then $b(G) \leq 1+d$. This is at most $p^{d / 2}$ unless $p=2$ and $2 \leq d \leq 5$. In particular $b(G) \leq 6$ and $n \leq 32$.

This completes the proof of Corollary 1.3 .

\section{REFERENCES}

[1] M. Aschbacher and R.M. Guralnick, On abelian quotients of primitive groups, Proc. Amer. Math. Soc. 107 (1989), no. 1, 89-95.

[2] L. Babai, On the order of uniprimitive permutation groups, Ann. of Math. (2) 113 (1981), 553-568.

[3] L. Babai, On the order of doubly transitive permutation groups, Invent. Math. 65 (1981/82), 473-484.

[4] C. Benbenishty, On actions of primitive groups, Ph.D. thesis, Hebrew University, Jerusalem, 2005.

[5] C. Benbenishty, J.A. Cohen and A.C. Niemeyer, The minimum length of a base for the symmetric group acting on partitions, European J. Combin. 28 (2007), 1575-1581.

[6] A. Bochert, Über die Transitivitätsgrenze der Substitutionengruppen, welche die alternirende ihres Grades nicht enthalten, Math. Ann. 33 (1889), 572-583.

[7] T.C. Burness, On base sizes for actions of finite classical groups, J. Lond. Math. Soc. (2) 75 (2007), $545-562$.

[8] T.C. Burness, R.M. Guralnick and J. Saxl, On base sizes for symmetric groups, Bull. Lond. Math. Soc. 43 (2011), 386-391.

[9] T.C. Burness, R.M. Guralnick and J. Saxl, On base sizes for algebraic groups, J. Eur. Math. Soc. (JEMS) 19 (2017), no. 8, 2269-2341.

[10] T.C. Burness, M.W. Liebeck and A. Shalev, Base sizes for simple groups and a conjecture of Cameron, Proc. Lond. Math. Soc. (3) 98 (2009), 116-162.

[11] T.C. Burness, E.A. O'Brien and R.A. Wilson, Base sizes for sporadic simple groups, Israel J. Math. 177 (2010), 307-333.

[12] T.C. Burness and Á. Seress, On Pyber's base size conjecture, Trans. Amer. Math. Soc. 367 (2015), $5633-5651$.

[13] H. Duyan, Z. Halasi and A. Maróti, A proof of Pyber's base size conjecture, arXiv:1611.09487, Submitted for publication.

[14] J.B. Fawcett, The base size of a primitive diagonal group, J. Algebra 375 (2013), 302-321.

[15] The GAP Group, Groups, Algorithms, and Programming, 2014, (http://www.gap-system.org).

[16] M. Giudici, M.W. Liebeck, C.E. Praeger, J. Saxl and P.H. Tiep, Arithmetic results on orbits of linear groups, Trans. Amer. Math. Soc. 368 (2016), 2415-2467.

[17] D. Gluck, Á. Seress, A. Shalev, Bases for primitive permutation groups and a conjecture of Babai, J. Algebra 199 (1998), 367-378.

[18] R.M. Guralnick, A. Maróti, L. Pyber, Normalizers of primitive permutation groups, Adv. Math. 310 (2017), 1017-1063.

[19] R.M. Guralnick and J. Saxl, Generation of finite almost simple groups by conjugates, J. Algebra 268 (2003), 519-571.

[20] Z. Halasi, On the base size for the symmetric group acting on subsets, Studia Sci. Math. Hungar. 49 (2012), 492-500.

[21] P. Kleidman and M. W. Liebeck, The subgroup structure of the finite classical groups, LMS Lecture Note Series, 129. Cambridge University Press, Cambridge, 1990.

[22] M. Lee and M.W. Liebeck, Bases of quasisimple linear groups, preprint.

[23] M.W. Liebeck, On minimal degrees and base sizes of primitive permutation groups, Arch. Math. (Basel) 43 (1984), 11-15.

[24] M.W. Liebeck, C.E. Praeger and J. Saxl, On the O'Nan-Scott theorem for finite primitive permutation groups, J. Austral. Math. Soc. Ser. A 44 (1988), 389-396.

[25] M.W. Liebeck and A. Shalev, Simple groups, permutation groups, and probability, J. Amer. Math. Soc. 12 (1999), 497-520. 
[26] M.W. Liebeck and A. Shalev, Bases of primitive linear groups, J. Algebra 252 (2002), 95-113.

[27] M.W. Liebeck and A. Shalev, Bases of primitive linear groups II, J. Algebra 403 (2014), 223-228.

[28] A. Maróti, On the orders of primitive groups, J. Algebra 258 (2002), no. 2, 631-640.

[29] C.E. Praeger, The inclusion problem for finite primitive permutation groups, Proc. London Math. Soc. (3) 60 (1990), 68-88.

[30] L. Pyber, On the orders of doubly transitive permutation groups, elementary estimates, J. Combin. Theory Ser. A 62 (1993), 361-366.

[31] L. Pyber, Asymptotic results for permutation groups, Groups and computation, DIMACS Ser. Discrete Math. Theoret. Comput. Sci. 11 (ed. L. Finkelstein and W. M. Kantor) Amer. Math. Soc. Providence RI 1993, pp. 197-219.

[32] Á. Seress, The minimal base size of primitive solvable permutation groups, J. London Math. Soc. 53 (1996), 243-255.

[33] J. Siemons and Z. Zalesskii, Intersections of matrix algebras and permutation representations of PSL (n,q), J. Algebra 226 (2000), 451-478.

Department of Algebra and Number Theory, EÖtvös University, PÁzmány Péter sétány 1/C, H-1117, Budapest, Hungary and Alfréd Rényi Institute of Mathematics, Hungarian Academy of Sciences, ReÁltanoda utca 13-15, H-1053, Budapest, Hungary

E-mail address: zhalasi@cs.elte.hu and halasi.zoltan@renyi.mta.hu

Department of Mathematics, Imperial College, London SW7 2BZ, United Kingdom

E-mail address: m.liebeck@imperial.ac.uk

Alfréd Rényi Institute of Mathematics, Hungarian Academy of Sciences, Reáltanoda utCa 13-15, H-1053, Budapest, Hungary

E-mail address: maroti.attila@renyi.mta.hu 